\title{
Obscurin Depletion Impairs Organization of Skeletal Muscle in Developing Zebrafish Embryos
}

\author{
Maide Ö. Raeker ${ }^{1}$ and Mark W. Russell ${ }^{1,2}$ \\ ${ }^{1}$ Department of Pediatrics and Communicable Diseases, University of Michigan, Ann Arbor, MI 48109, USA \\ ${ }^{2}$ Cellular and Molecular Biology Graduate Program, University of Michigan, Ann Arbor, MI 48109, USA
}

Correspondence should be addressed to Mark W. Russell, mruss@med.umich.edu

Received 2 May 2011; Revised 10 July 2011; Accepted 27 July 2011

Academic Editor: Robert J. Bloch

Copyright ( 2011 M. Ö. Raeker and M. W. Russell. This is an open access article distributed under the Creative Commons Attribution License, which permits unrestricted use, distribution, and reproduction in any medium, provided the original work is properly cited.

\begin{abstract}
During development, skeletal myoblasts differentiate into myocytes and skeletal myotubes with mature contractile structures that are precisely oriented with respect to surrounding cells and tissues. Establishment of this highly ordered structure requires reciprocal interactions between the differentiating myocytes and the surrounding extracellular matrix to form correctly positioned and well-organized attachments from the skeletal muscle to the bony skeleton. Using the developing zebrafish embryo as a model, we examined the relationship between new myofibril assembly and the organization of the membrane domains involved in cell-extracellular matrix interactions. We determined that depletion of obscurin, a giant muscle protein, resulted in irregular cell morphology and disturbed extracellular matrix organization during skeletal muscle development. The resulting impairment of myocyte organization was associated with disturbance of the internal architecture of the myocyte suggesting that obscurin participates in organizing the internal structure of the myocyte and translating those structural cues to surrounding cells and tissues.
\end{abstract}

\section{Introduction}

Skeletal muscle function is dependent upon stable connections from the contractile elements that generate force to the bony skeleton that translates that force into movement. Efficient transmission of that contractile force is enabled by a highly organized arrangement of support structures both within and around the actin-myosin array. Through these supporting elements, the actin-myosin array within the cell is directly connected to the extracellular matrix (ECM). These connections occur both along the axis of contraction at the myotendinous junction (MTJ), where the muscle inserts into a fibrous tendon that attaches to the bony skeleton, and radially at specialized membrane domains like the costameres $[1,2]$, where the muscle attaches to surrounding fibrous sheaths called the endomysium and the perimysium. The importance of muscle-ECM connections to muscle structure and function is evident from the number of myopathies and muscular dystrophies, including Duchenne muscular dystrophy, that result from disturbance of the multiprotein complexes that support the linkage [3].
The primary site of force transmission from the muscle to the ECM, and ultimately to the bony skeleton, occurs at the myotendinous junctions. Despite the importance of the MTJ to muscle function, relatively little is known about how it is formed. What is known has been derived from studies in zebrafish where visualization of embryos during development has allowed direct observation of the dynamic process of MTJ formation and skeletal muscle development [4]. As with other vertebrates, truncal skeletal muscle in the zebrafish is primarily derived from repeating structures called somites. The mesenchymal cells of the somite give rise to the myotome, which will differentiate into skeletal myocytes that span the length of the somite and insert into the fibrous sheaths that form at the anterior and posterior somite boundaries [5]. These muscle-matrix connections are the structural and functional equivalents of the MTJs that form in higher vertebrates [6].

Previous studies have determined that Eph/Ephrin signaling initiates integrin clustering along the nascent somite border concomitant with the earliest indication of border 
formation and shortly after that fibronectin matrix is assembled. Integrins are cell-matrix adhesion molecules that form heterodimeric transmembrane units which bind to components of the extracellular matrix including collagen and fibronectin. Laminin is secondarily deposited at the somite boundary where it serves as a ligand for $\alpha$-dystroglycan of the dystrophin-associated glycoprotein complex during progressive maturation of the MTJ [7]. A zebrafish model of Duchenne muscular dystrophy, the sapje mutant which has a truncating mutation of dystrophin, forms MTJs at the somite boundary but these attachments are unstable, leading to muscle detachment and myocyte death [8].

The finding of MTJ instability in the zebrafish model of DMD suggested that this may be an important mechanism of muscle injury in patients with this disorder. This was a somewhat unexpected finding as MTJs had been difficult to study in DMD patients or mammalian animal models which had focused on the effects of dystrophin loss on the composition and function of lateral muscle-ECM attachments within the costameres [9]. It also indicated that the formation and composition of myofibril-membrane attachments may be similar whether they form along the longitudinal axis of the cell at the MTJ or along the lateral aspect of the myocyte, where dystrophin and the dystrophin-associated glycoprotein complex are enriched. The lateral connections occur primarily at the costameres overlying the sarcomere at the level of the Z disk [2]. As with the MTJ, the transmembrane adhesive contacts at the costameres include the integrinbased and dystrophin-associated glycoprotein complexes. These transmembrane systems are linked to each other and to the actin cytoskeleton by an array of cytoskeletal adaptors including vinculin, talin, ILK, and filamin. The adaptor proteins regulate the adhesive properties of the connections and allow the scaffolding of signaling molecules at these sites.

What is not well understood is how these myocyte-ECM attachments are positioned and assembled relative to the underlying myofibrils, and whether or not these processes are similar for axial (MTJ) and radial (costameric) connections. In this paper, we used a zebrafish morphant phenotype with impaired MTJ formation and skeletal muscle disorganization [10] to examine the elements required for muscle-ECM attachment and for the coordination of myofibril organization across adjacent myocytes. We determined that the giant structural and signaling protein obscurin has an important role in promoting the organization of the extracellular matrix at the terminal ends of the myocyte and in supporting myofibril-membrane interactions that lead to alignment of myofibrils both within and across myocytes. As myocyteextracellular matrix connections have critical roles in force transduction defining the processes that promote and sustain these connections will have important implications for the understanding the pathogenesis of, and developing new treatment strategies for, a range of myopathies and muscular dystrophies.

\section{Methods}

2.1. Zebrafish Maintenance and Breeding. Wild-type adult zebrafish were maintained as described previously [10, 11].
2.2. Morpholino Oligonucleotide Microinjection. The obscurin A antisense morpholino (MO2 from [12]) targeting the $5^{\prime}$ UTR site and a standard control morpholino (5' CCTCTTACCTCAGTTACAATTTATA-3') were designed by GENE TOOLS, LLC (Philomath, Ore, USA). The MOs were dissolved to a stock concentration of $3 \mathrm{mM}$ in distilled water and diluted to 1.5 and $3 \mathrm{ng} / \mathrm{nL}$. A $2.5 \mathrm{~nL}$ aliquot of diluted morpholinos were injected into 1-4-cell-stage embryos using a nanoliter 2000 injector (World Precision Instruments). A Leica MZ16F dissecting microscope equipped with DFC340 FX camera was used to visualize the embryos.

2.3. mRNA Injections. A lyn-GFP (pCS-memb-mCherry + lyn-eGFP) construct, kindly provided by Dr. Phil Elks (University of Sheffield), was linearized with NotI and capped mRNA was generated using the mMESSAGE mMACHINE SP6 kit (Ambion, Austin, Tex, USA). The lyn-GFP mRNA was injected at an amount of $62.5 \mathrm{pg} / \mathrm{embryo}$ alone and together with obscurin A morpholino. A pCS2 + itga5emGFP construct, kindly provided by Dr. Scott Holley (Yale University), was linearized with NotI and transcribed with SP6 polymerase using the mMESSAGE mMACHINE SP6 kit from Ambion. The itga5-GFP mRNA was injected at a $250 \mathrm{pg} / \mathrm{embryo}$ amount alone and together with obscurin A morpholino.

\subsection{Wholemount Immunostaining and In Situ Hybridization.} Embryos were fixed for $6 \mathrm{hr}$ with $4 \%$ paraformaldehyde (PFA) in PBS and labeled with the following antibodies: obscurin (directed at the $\mathrm{C}$ terminal region where the small ankyrins bind (kindly provided by Dr. Robert Bloch, University of Maryland)) $1: 10$, fibronectin (Sigma) $1: 50$, laminin (Sigma) 1:50, F59 (Developmental Hybridoma Bank, University of Iowa) $1: 10, \alpha$-actinin (Sigma) $1: 100$, vinculin (Sigma) $1: 50$, talin (Sigma) $1: 50$, dystrophin (Clone MANDRA1, Sigma) $1: 50$, and $\alpha$-dystroglycan (Millipore) $1: 20$. Secondary antibodies Alexa 640, Alexa 594, and Alexa 488 from Molecular Probes were diluted to $1: 150$ to reveal staining. Embryos were mounted onto slides using ProLong Gold Antifade Reagent (Molecular Probes) and visualized on an Olympus FV-500 confocal microscope. Digoxigenin-labeled antisense RNA probes were synthesized from cDNA clones of MyoD, Notc5, Notch6, and obscurin A by in vitro transcription using T3 and T7 polymerases and DIG RNA labeling kit (Boehringer-Mannheim). Embryos were fixed in 4\% PFA for 5 hours at room temperature and subjected to in situ hybridization with the above RNA probes. Stained embryos were visualized on an Olympus BX-51 light microscope.

2.5. RNA Isolation and RT-PCR. To determine the onset of obscurin A expression, total RNA was isolated from uninjected embryos at 3, 4.5, 6, 8.5, 10, 11.5, 13, 15, 24, 33, and 72 hours after fertilization (hpf) using Qiagen RNeasy Mini Kit (Qiagen Inc., USA). RNA concentration and purity was determined by optical density reading at $260 \mathrm{~nm}$ and the ratio of 260/280 $\mathrm{nm}$ absorbance, respectively. For all time points, with the exception of $8.5 \mathrm{hpf}$, a total of $75 \mathrm{ng}$ RNA was used in a $25-\mu \mathrm{L}$ reaction volume to synthesize 


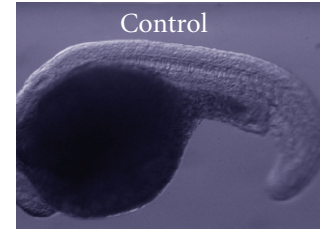

(a)

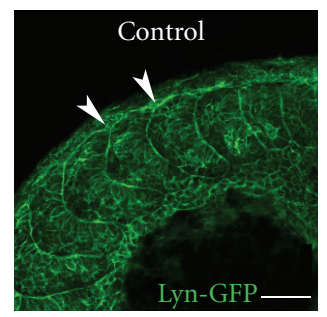

(c)

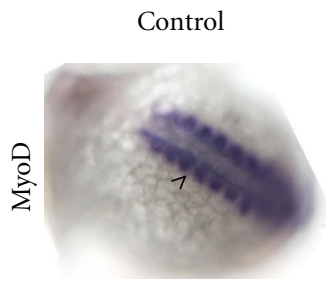

(e)

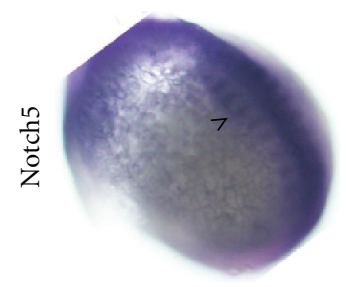

(g)

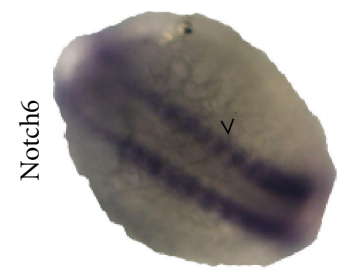

(i)

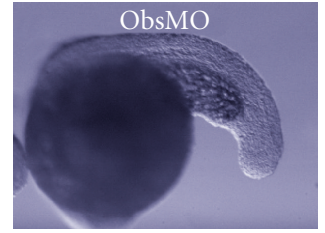

(b)

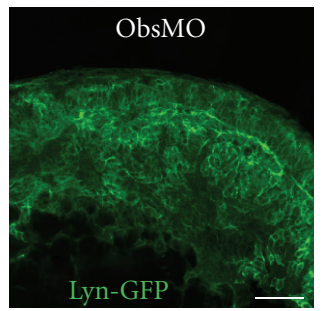

(d)

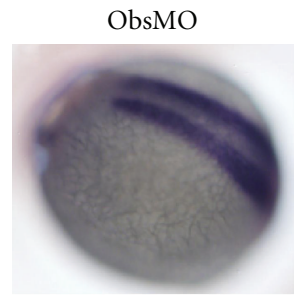

(f)

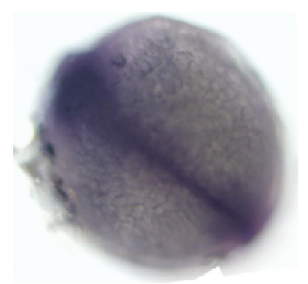

(h)

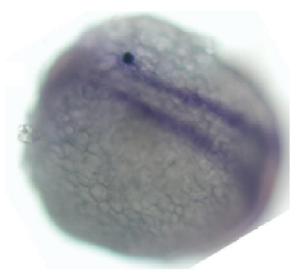

(j)
Figure 1: Obscurin A depletion results in abnormalities of myotome development. (a, b) Compared to control embryos at $20 \mathrm{hpf}$, an obscurin-A-depleted embryo (ObsMO) demonstrates shortening of the tail and indistinct somite boundaries. The skeletal muscle abnormalities are associated with irregularities of cell morphology as noted by cell membrane localization of lyn-GFP (c, d). Note that in control embryos, (c) lyn-GFP accumulates at the somite boundary (arrowheads). In morphant embryos (d), distinct somite boundaries are difficult to identify. (e-j) RNA in situ hybridization using riboprobes for MyoD, Notch5, and Notch6 in control (e)-(i) and obscurin A (f), (h), (j) morphant embryos demonstrate maintained expression but diminished periodicity of these somite markers suggesting preserved myocyte commitment but impaired somite organization in the setting of obscurin depletion. Scale bars are $50 \mu \mathrm{m}$ (a)-(d) and $500 \mu \mathrm{m}(\mathrm{e})-(\mathrm{j})$.
cDNA using the iScript One-Step RT-PCR kit with SYBR green (Bio-Rad Laboratories, Hercules, Calif, USA). Due to a very low RNA concentration in the isolate, about $15 \mathrm{ng}$ of RNA was used in the reaction mix for $8 \mathrm{hpf}$ time point. A $350 \mathrm{bp}$ (forward primer, 5'-GCCTGACCTGCGCGATTGTGA; reverse primer, 5' -GAGCGCTGGGAGAGGGAGGAC) and a $250 \mathrm{bp}$ (forward primer, 5'GCACCAAACCCACCAAATCCA; reverse primer, 5'GCGCCCTCCTCATCCACACG), zebrafish obscurin A cDNA fragments were amplified by PCR. As a control, two reactions were prepared: one without RNA and one without reverse transcriptase. Zebrafish $\beta$-Actin (forward primer, $5^{\prime}$-CCGTGAACATCAAGGAGAAGCT; reverse primer, 5'-TCGTGGATACCGCAAGATTCC) was used as an internal control.

2.6. Electron Microscopy. Electron microscopy was performed as previously described [10]. Briefly, embryos at $72 \mathrm{hpf}$ were fixed with $2.5 \%$ glutaraldehyde and $2.0 \%$ paraformaldehyde and postfixed in 1\% osmium tetroxide. After washing, they were stained with saturated uranyl acetate and embedded in Epon 812. Ultrathin sections were prepared and stained with saturated uranyl acetate and lead citrate. The sections were examined with a Philips CM100 transmission electron microscope, at an accelerating voltage of $60 \mathrm{kV}$. The distance between the myofibrils in adjacent myocytes was measured from the transverse sections at the minimal distance between the two myofibrils. $\mathrm{Z}$ disk offset was measured in superficial myofibrils from two adjacent myocytes. In both control- and obscurin-depleted embryos, a minimum of 30 myocyte pairs were measured at 24 and 72 hpf.

\section{Results}

3.1. Obscurin A Depletion Phenotype. To examine the relationship between the myofibril and the organization of specialized membrane compartments during muscle development, we used a previously described morphant model [10] in which depletion of a myofibrillar protein, obscurin $[13,14]$, led to the impairment of the organization of the myotendinous junction, which is formed through the interactions of specialized membrane domain of the myocyte with the extracellular matrix. As previously noted, obscurin A morphant embryos were shorter, with marked abnormalities in tail and skeletal muscle morphology (Figure 1). These included both abnormalities of tissue architecture (irregular and deficient somite boundaries) and cellular organization (impaired SR organization, increased incidence of breaks in the transverse alignment of adjacent myofibrils, and irregularities of myofibril bundling) [10].

In obscurin morphant embryos, defects were noted in the developing myotome prior to the onset of myofibrillogenesis. Somite markers MyoD, Notch5, and Notch6 were expressed in both the control and obscurin-A-depleted embryos, but the periodicity of their expression, namely, the absence of expression at the future somite boundaries, was impaired in the obscurin A morphants (Figure 1). Since this defect in myotome organization would suggest an important role 
for obscurin A very early in skeletal muscle development, prior to the onset of myofibrillogenesis, we sought to precisely define the onset and localization of obscurin A expression very early in embryogenesis. Using RT-PCR, RNA in situ hybridization and whole mount immunostaining, we determined that obscurin A is expressed very early during somitogenesis and that it first localizes to the newly forming somite boundaries, see supplementary materials avaliable at doi:10.1155/2011/479135 (Supplemental Figure 1).

To examine the role of obscurin $\mathrm{A}$ in promoting the changes in cell morphology that permit the formation of the somite boundary, control and obscurin A morphant embryos were injected with lyn-GFP mRNA and fixed at 18, 24 , and $48 \mathrm{hpf}$. Lyn is a member of the Src family of receptor tyrosine kinases that is associated with cell surface receptors [15] and has been used to help track changes in cell morphology during development [16]. Lyn localized to the cell membranes of the developing myoblasts and accumulated at the nascent somite boundaries (Figure 2(a)). In the younger somites, myoblasts (future fast-twitch skeletal muscle) were rounded and began to elongate after the development of the somite boundary (Figure 2(b)), eventually spanning the distance between the anterior and posterior boundaries and inserting into the fibrous matrix forming at the boundary. In obscurin-A-depleted embryos, fewer nascent boundaries could be identified (Figure 2(d)). In the more severely affected embryos, the cells remained rounded and no defined boundaries were noted (Figure $2(\mathrm{~g})$ ). In the mildly affected embryos, irregular boundaries did form (Figure 2(e)) and were associated with local elongation of the adjacent cells. In the absence of a defined boundary, cells initially remained rounded (Figures $2(\mathrm{e})$ and $2(\mathrm{~h})$ ), eventually elongating in the more mature somites (Figures 2(e) and 2(f)). This suggests that somite boundary formation (and the associated extracellular matrix organization) promotes cell elongation but that cell elongation can progress in the absence of the somite boundaries potentially as a result of positional cues from surrounding cells.

3.2. Clustering of Integrins. Previous studies have noted that integrin clustering precedes fibronectin matrix accumulation at the nascent somite boundary [17] and helps facilitate tissue remodeling during development by translating cell contact-mediated signals into cellular cytoskeletal reorganization. Therefore, we examined the localization of $\alpha 5$ integrin, which participates in somite boundary formation $[18,19]$ during early skeletal muscle differentiation. Using a GFP-tagged $\alpha 5$-integrin expression construct, we noted that $\alpha 5$-integrin clustering at the nascent somite boundary closely preceded organization of the fibronectin matrix and elongation of the cells at the developing boundary (Figure 3). As noted previously, depletion of the giant protein obscurin with a morpholino targeting the $5^{\prime}$ untranslated region of the obscurin A isoform resulted in marked abnormalities of somite boundary and MTJ formation [10]. This provided unique opportunity to observe the progression of MTJ maturation and define the elements required for the process. Depletion of obscurin revealed that integrin clustering, fibronectin organization, and cellular remodeling are closely interrelated processes. In response to obscurin depletion, integrin clustering was impaired resulting in diminished fibronectin matrix organization and abnormalities of cell morphology. In those regions with preserved integrin clustering, a fibronectin matrix was organized and cells began to elongate. In the absence of integrin clustering, there was no organization of a fibronectin matrix and cells remained rounded. This is consistent with prior studies which have proposed that cytoskeletal actin reorganization and cellular remodeling during myoblast differentiation are dependent on the organization of the fibronectin matrix [20]. The findings also suggest that obscurin, which is expressed at very low levels prior to myoblast differentiation, may have a role in supporting the early cellular events that lead to apical clustering of integrins.

\subsection{Organization of the Fibronectin Matrix and Its Rela-} tionship to Nascent Myofibrils. Given the potential role of fibronectin in determining myocyte morphology and skeletal muscle organization [20], the relationship between fibronectin organization and new myofibril assembly was examined during early skeletal muscle differentiation. Fibronectin was organized first immediately adjacent to the notochord, prior to the appearance of a clear striated pattern of myosin heavy chain or $\alpha$-actinin immunostaining in assembling myofibrils of slow-twitch skeletal muscle. Alphaactinin appeared first as closely and irregularly spaced punctuate spots that were gradually organized into distinct $\mathrm{Z}$ disk structures in the more mature somites (data not shown). In contrast, very early in myofibrillogenesis, myosin heavy chain II (sarcomeric myosin) was diffusely distributed along the length of the myofibril (Figure 4). As myofibril assembly progressed, myosin becomes restricted to nascent $\mathrm{A}$ bands before demonstrating the double-banded pattern with mid-A-band clearing characteristic of mature F59 antibodystaining pattern which recognizes an epitope within the myosin motor domain. In response to obscurin depletion, there was a normal progression of $\mathrm{M}$ band organization despite the noted irregularities of fibronectin matrix deposition and myocyte morphology. Of note, in response to obscurin depletion, there was delayed bundling of nascent myofibrils into larger units even at relatively advanced stages of myofibrillogenesis, A bands from adjacent myofibrils remained spatially distinct. The delayed structural integration of adjacent myofibrils is consistent with the abnormalities in the lateral alignment of myofibrils that was previously noted in response to obscurin depletion [10]. Therefore, fibronectin matrix organization precedes the formation of mature, striated myofibrils, and despite the effects of obscurin on the fibronectin matrix, the progression of thick filament organization proceeds to structural maturity.

3.4. Deposition of Laminin. Laminin deposition at the MTJ closely followed fibronectin organization and persisted after fibronectin downregulation (Figure 5). In embryos subject to obscurin depletion, fibronectin matrix organization was severely reduced. In contrast, while laminin deposition was irregular, it did accumulate normally in those regions where fibronectin was detected. This indicates that fibronectin 


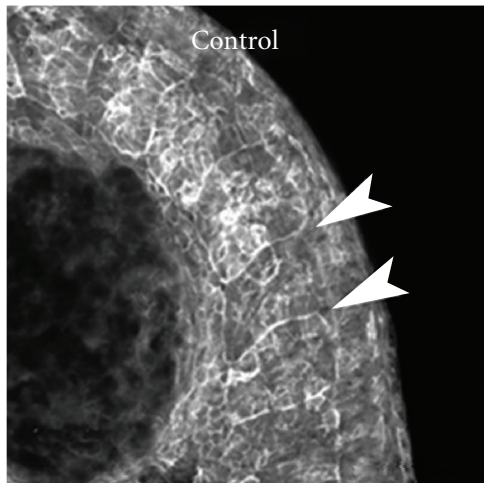

(a)

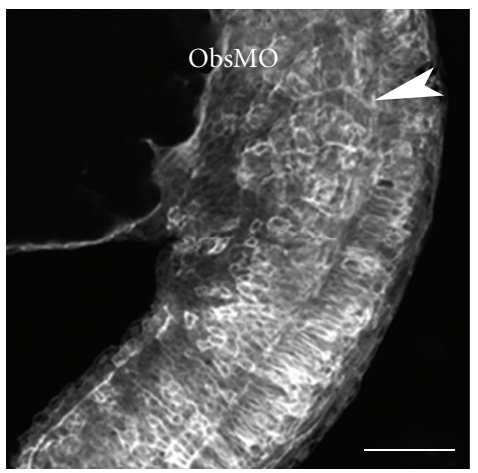

(d)

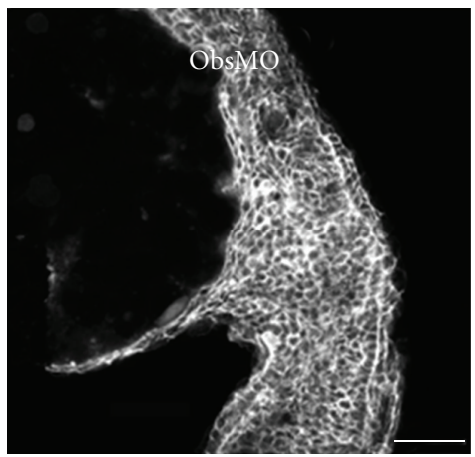

(e)

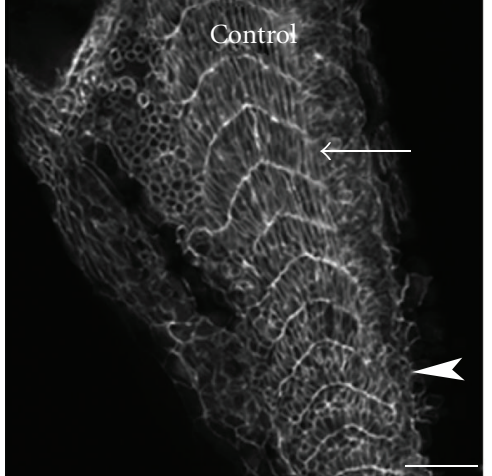

(b)

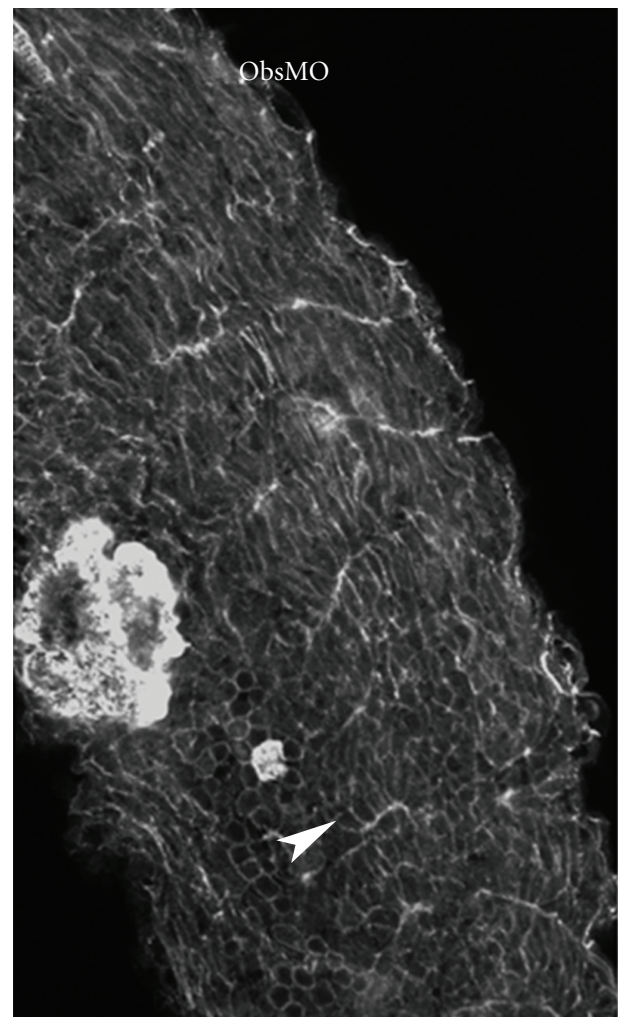

(f)

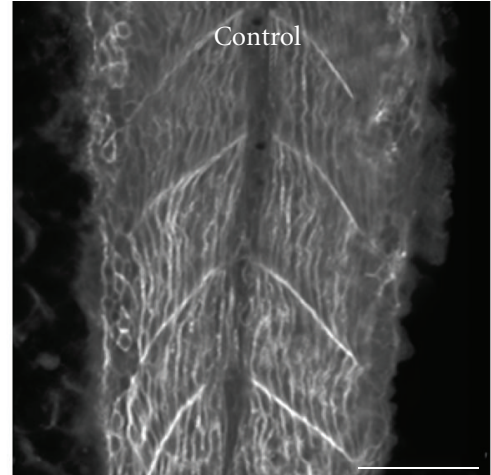

(c)

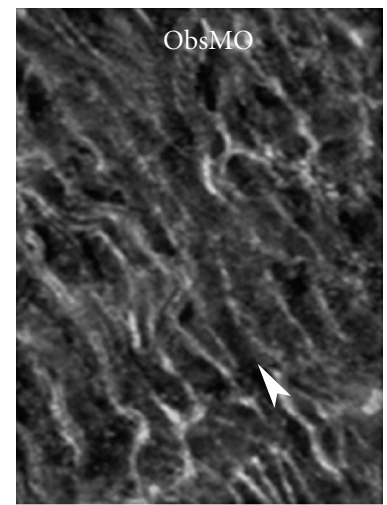

(g)

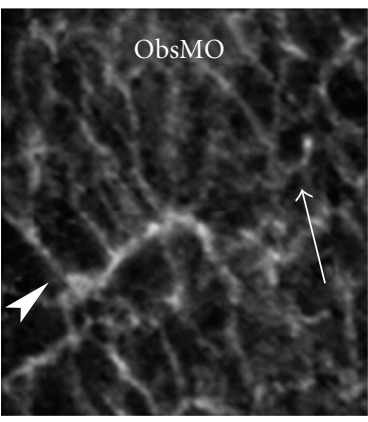

(h)

Figure 2: Obscurin A depletion results in abnormalities in the tissue architecture of skeletal muscle. Control (a)-(c) and obscurin A morphant (d)-(h) embryos were injected with a mRNA encoding for lyn-GFP and fixed at 18 (a), (d), (g), 24 (b), (e)-(h), and 48 (c) hpf. (a) Lyn is incorporated into the membrane and accumulates along the somite boundaries as they form (arrowheads). (b) As the myocytes mature, they transition from rounded morphology (arrowhead) and elongate (arrow) to span the length of the developing myotome to insert into the ECM at the MTJ. (c) By 48 hpf, all the fast-twitch skeletal myocytes have elongated. (d) In response to obscurin depletion, only rare somite boundaries (arrowhead) were noted. (e) In a more severely affected obscurin morphant embryo, no nascent somite boundaries are detected and the myoblasts remain rounded. (f) As development proceeds in a mildly affected obscurin morphant embryo, patchy development of nascent MTJs reveals a close relationship between apical lyn accumulation and cell elongation (arrowhead). (g) In the more mature somites, myocyte elongation proceeds in the absence of apical lyn clustering (arrowheads). (h) In a younger somite, myoblast elongation (arrowhead) preferentially occurs at the site of apical lyn clustering while other cells remain rounded (arrow). Scale bars are $50 \mu \mathrm{m}$ (a)-(f) and $10 \mu \mathrm{m}$ (g), (h).

matrix organization supports and may be required for laminin deposition as has been previously suggested [5]. Furthermore, although obscurin participates in integrin clustering and fibronectin matrix organization, the subsequent recruitment of laminin to areas of fibronectin accumulation is likely to be independent of obscurin A function. Laminin deposition preceded formation of striated myofibrils in fast-twitch skeletal muscle. In the regions of reduced, 


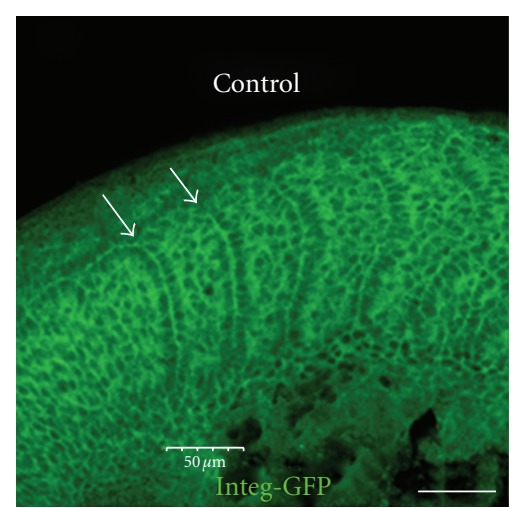

(a)

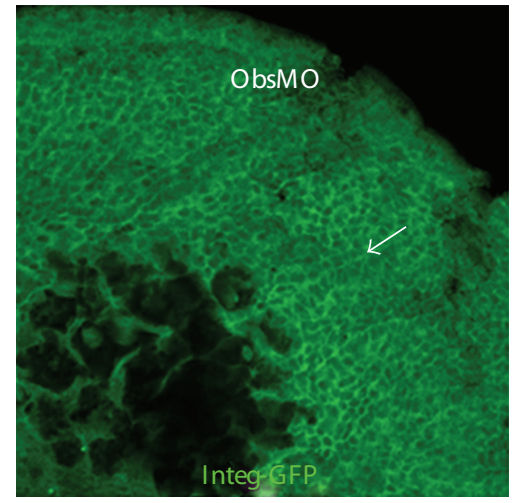

(c)

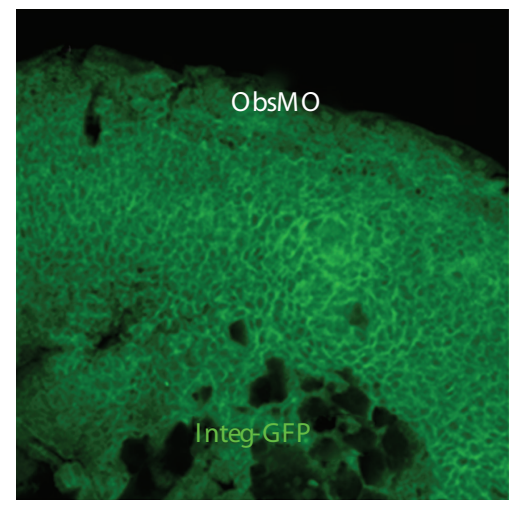

(e)

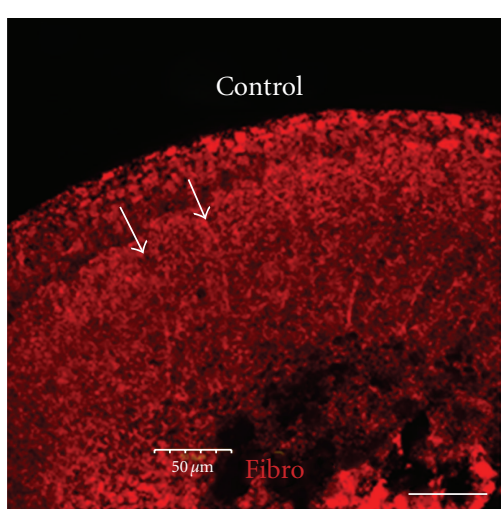

(b)

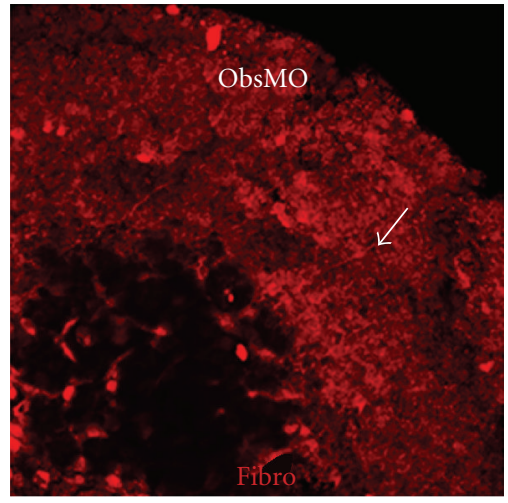

(d)

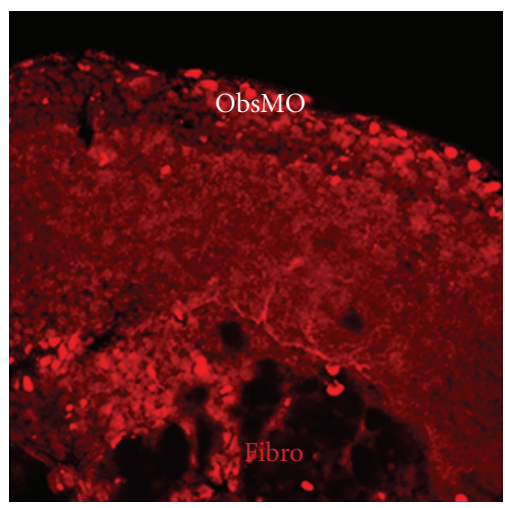

(f)

FIGURE 3: Integrin clustering is impaired in zebrafish embryos depleted of obscurin A. Zebrafish embryos were injected with GFP- $\alpha 5$ integrin mRNA and then immunolabeled with anti-GFP (green: (a), (c), (e)) and antifibronectin (red: (b), (d), (f)) antibodies at 18 hours after fertilization (hpf). (a), (b) In control embryos, fibronectin matrix is organized at sites where $\alpha 5$-integrin has clustered along a nascent somite boundaries (arrows). (c)-(e) In mildly affected (c), (d) and more markedly affected (e), (f) obscurin-A-depleted embryos, there is reduced apical clustering of integrins along nascent boundaries, although in the few instances where there is significant integrin clustering, fibronectin is organized ((c), (d): arrows). Scale bars are $50 \mu \mathrm{m}$.

fibronectin/laminin deposition, myofibrillogenesis appeared to be delayed, as regions with more abundant laminin deposition demonstrated more rapid development of mature myofibrils. However, by 72 hours, even those regions with markedly reduced laminin deposition demonstrated mature myofibril development.

3.5. Recruitment of Intracellular Adaptors to the Cell-ECM Adhesion Complex. To determine the order of incorporation of other components of the adhesion complex and examine their relative distribution to MTJ and costameric sites, immunolocalization of talin and vinculin, components of the adherens junctions, was performed. Both talin and vinculin localized to the MTJ nearly concurrently with the timing of laminin deposition (Figure 6). However, as skeletal muscle differentiation proceeded their localization, patterns were distinct. Talin was noted to localize very early to membrane domains, and potentially to cytoskeletal structures, 


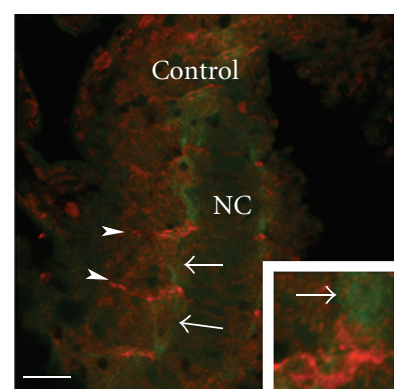

(a)

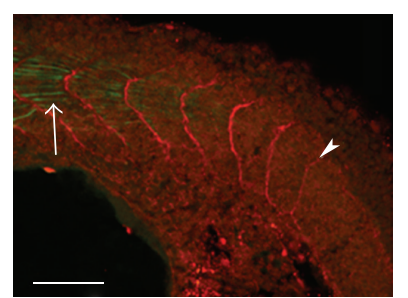

(b)

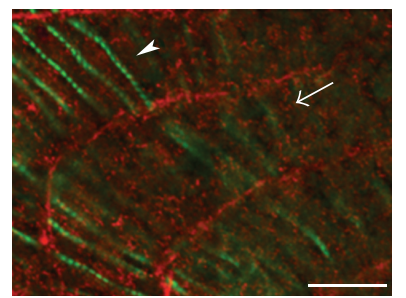

(c)

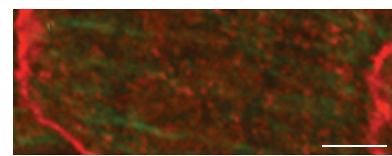

(d)

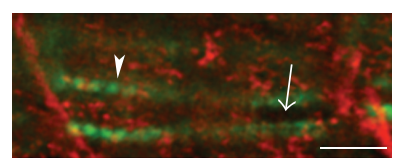

(e)

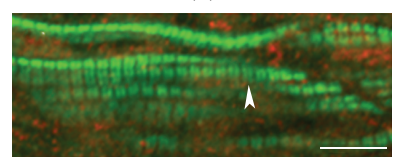

(f)

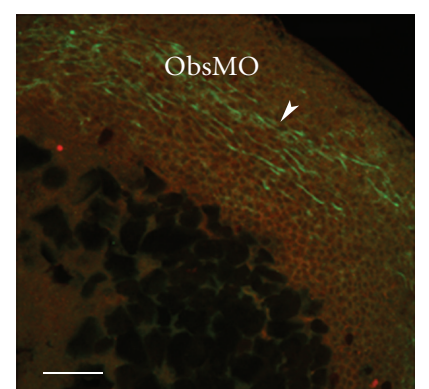

(g)

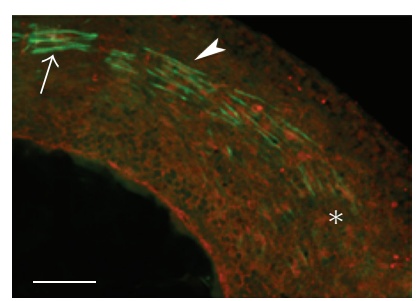

(h)

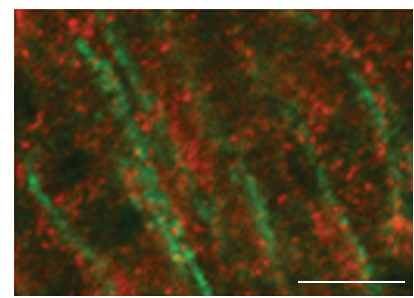

(i)

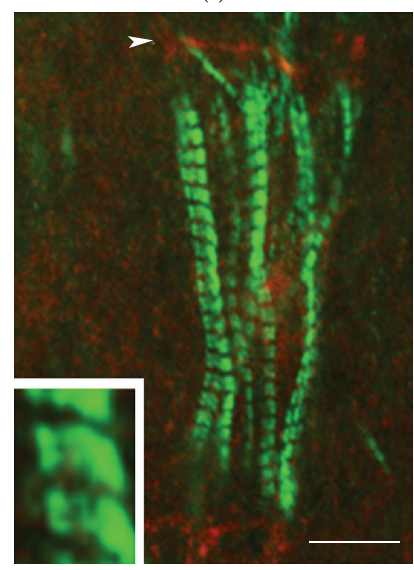

(j)

Figure 4: Organization of the fibronectin matrix precedes new myofibril assembly. Zebrafish embryos at 14 (a), 20 (b)-(e), (g), 24 (f), (h)(j) hpf were immunolabeled for fibronectin (red) and slow-twitch myosin heavy chain (F59 antibody) (green). (a) Fibronectin organizes next to the notochord (NC) at nascent somite boundaries (arrowheads). Myosin heavy chain is still diffusely localized (arrows: (a) and inset) as the fibronectin matrix is organizing. (b) In a control embryo, the fibronectin matrix (arrowhead) is organized at the somite boundary before assembly of mature myofibrils in slow-twitch skeletal muscle of the younger somites at the right of the panel. (c) At higher magnification, continuous bands of myosin staining (arrow) are noted extending between the organized fibronectin matrix at either end of the myocytes before organizing into a striated pattern (arrowhead). (d)-(f) As development proceeds, MHC localization transitions from a diffuse distribution (arrows) along new myofibrils to a distinct restriction to the A band (arrowheads) and finally to a mature "doublebanded" pattern ((f): arrowhead) with central clearing in the region of the future M band. By comparison, obscurin A depletion (g)-(j) affects fibronectin matrix organization and results in reduced organization of the MHC filaments. (g) - (j) Myofibril maturation can progress from immature myofibrils ((g), (h): arrowheads) to mature myofibrils ((h): arrow) even with a reduced and poorly organized fibronectin matrix (i). However, myofibrillogenesis proceeds more rapidly in regions with preserved fibronectin deposition ((j): arrowhead). Note the fibronectin organization at the terminal ends of the myofibrils (j). Lateral bundling of myofibrils appeared to be delayed in response to obscurin depletion as noted by the gaps between the A bands of neighboring but not yet fused myofibrils (inset of $(\mathrm{j})$ ). Scale bars are $50 \mu \mathrm{m}$ (b), (h), $20 \mu \mathrm{m}$ (a), and $10 \mu \mathrm{m}$ (c), (d), (e), (f), (i), (j). Insets in (a) and (j) are 3x magnification of the larger image. 


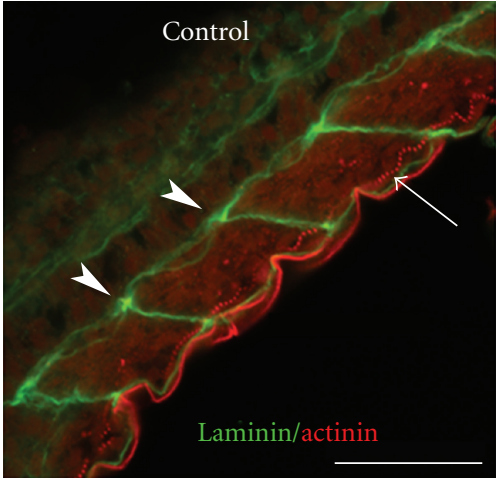

(a)

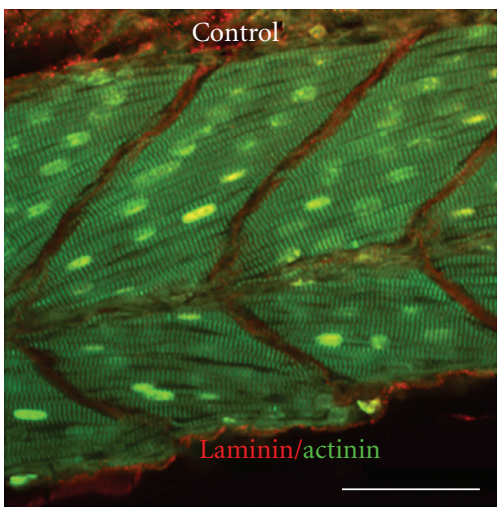

(c)

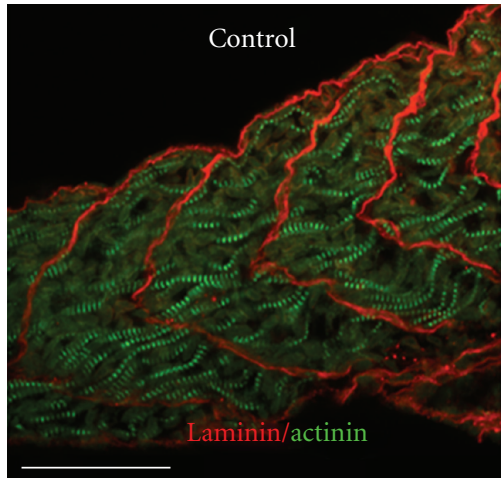

(b)

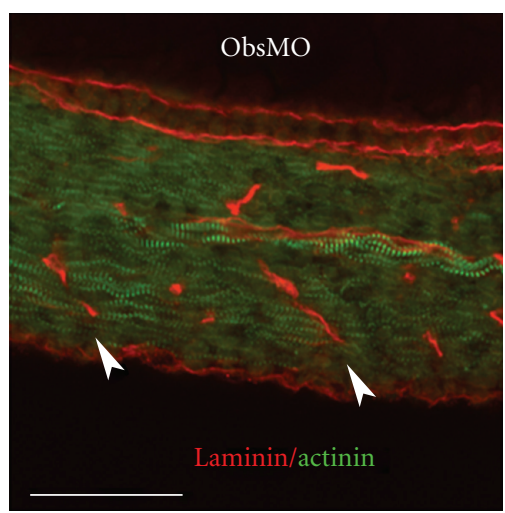

(d)

FIGURE 5: Laminin deposition precedes but is not a requirement for fast-twitch skeletal muscle myofibrillogenesis. Zebrafish embryos were immunolabeled for laminin ((a): green; (b), (c), (d): red) and $\alpha$-actinin ((a): red; (b), (c), (d): green) at 20 (a), 24 (b), (d), and 48 (c) hpf. (a) A mature laminin matrix has formed at the somite/myotome boundaries (arrowheads) and along the surface of the myotome prior to fast-twitch skeletal muscle myofibrillogenesis. By this time, slow-twitch skeletal muscle has migrated to its superficial location within the myotome (arrow). As fast-twitch skeletal muscle myofibrillogenesis progresses, laminin persists at the somite boundary at 24 (b) and 48 (c) hpf. In response to obscurin A depletion (d), laminin accumulates normally at the irregularly localized and incomplete MTJs (arrowheads). Fast-twitch skeletal muscle myofibrillogenesis is delayed in the regions lacking laminin deposition but does eventually proceed. Scale bars are $50 \mu \mathrm{m}$ (a), (b), (d) and $30 \mu \mathrm{m}$ (c).

overlying the $\mathrm{Z}$ disk. In contrast, vinculin did not demonstrate significant striated patterning by 72 hours afterfertilization and instead became progressively more concentrated at the MTJ. Vinculin patterning in the obscurin morphant embryos was irregular in distribution but normal in its accumulation at the MTJ. Likewise, talin localized normally to domains overlying the $\mathrm{Z}$ disk and to the MTJ in obscurin morphant embryos. However, there were areas of laminin deposition that lacked significant talin accumulation suggesting a potential delay or irregularity in adhesion complex assembly in response to obscurin A depletion.

\subsection{Localization of Dystrophin and $\alpha$-Dystroglycan Is Obscur-} in A Dependent. Dystrophin is a cytoskeletal protein that links the actin cytoskeleton to the membrane-bound dystrophin-associated glycoprotein complex (DGC). $\alpha$-DG, a member of DGC, is a cell surface receptor for various ECM molecules including laminins, agrin, and perlecan. Previous studies indicate that the DGC participates in the organization of, and attachment of the myocyte to, laminin on the cell surface. Since dystrophin and $\alpha$-DG are impor- tant components of the costameres and the myotendinous junctions, we examined their localization in control and obscurin A morphant embryos at $24 \mathrm{hpf}$ (Figure 7). In control morpholino-injected embryos, both dystrophin and $\alpha$-DG localized to the MTJ as has been previously noted $[21,22]$. In obscurin-A-depleted embryos, however, the localization of dystrophin is less distinct and $\alpha$-DG could not be detected at the somite boundaries. Since even the most severely affected obscurin A morphant embryos form irregular somite boundaries, the marked decrease in $\alpha$-DG staining suggested a specific destabilization of the DGC in the obscurin morphants.

\subsection{Obscurin A Depletion Results in Impaired Relationships} between the Myofibril and Overlying Sarcolemma. It has been postulated that ankyrin within the sarcolemma and the subsarcolemmal spectrin cytoskeleton are involved in establishing the membrane microdomains overlying the superficial myofibrils [23]. Since obscurin A interacts with both ankyrin within the sarcolemma $[12,24,25]$ and titin within the sarcomere $[14,26]$, it was a good candidate to link 


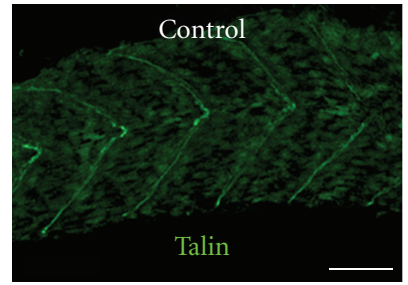

(a)

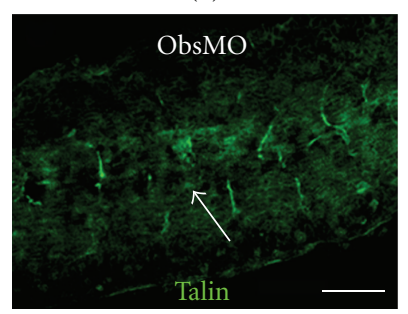

(c)

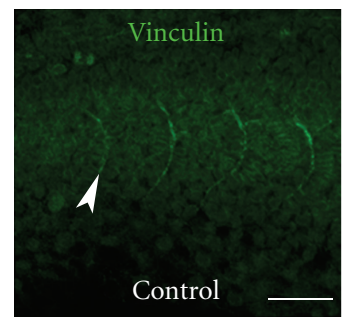

(h)

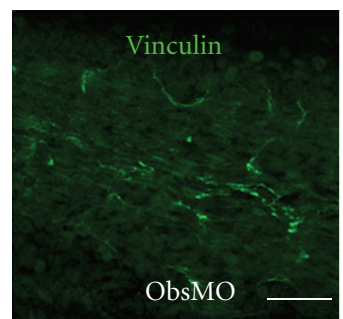

(k)

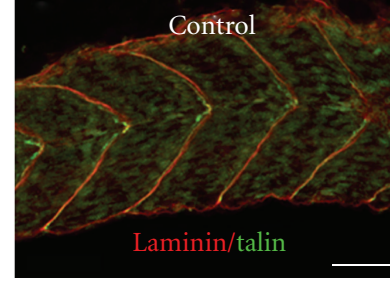

(b)

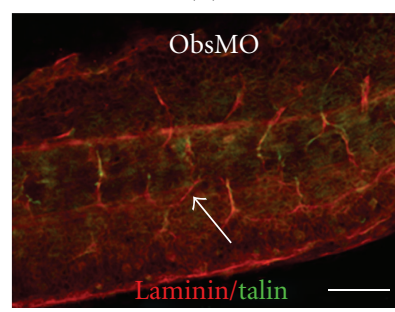

(d)

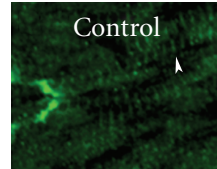

(e)

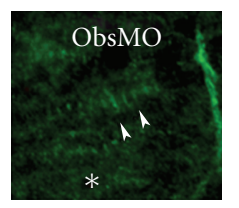

(f)

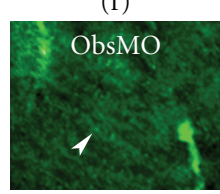

(g)

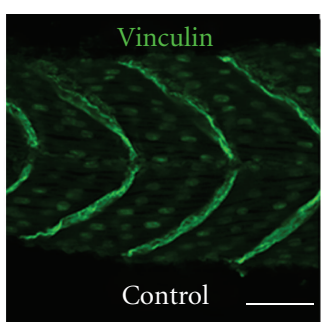

(i)

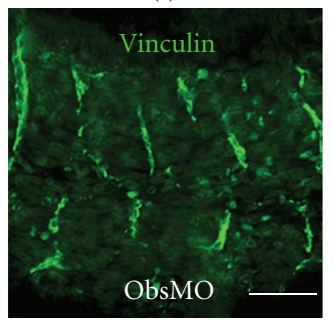

(1)

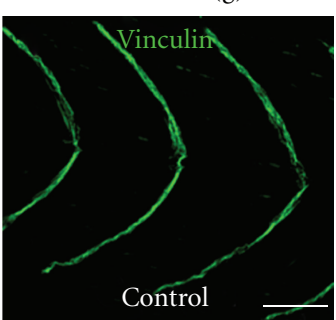

(j)

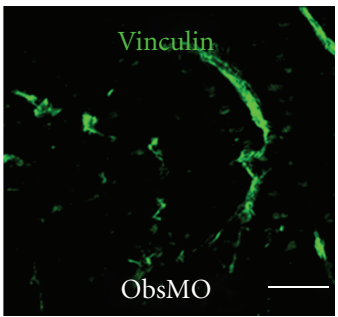

(m)

FIGURE 6: Overlapping localization of talin and vinculin during early skeletal muscle development. Control (a), (b), (e), (h)-(j) and obscurin A morphant (c), (d), (f), (g), (k)-(m) embryos were immunostained for talin ((a)-(g): green) and laminin ((b), (d): red) or vinculin ((h)(m): green) at 24 (a)-(h), (k), 48 (i), (l), or 72 (j), (m) hpf. (a) In a control embryo, talin localizes to the MTJ and overlying the myofibrils at $24 \mathrm{hpf}$. (b) At the MTJ, talin localizes immediately adjacent to laminin. (c), (d) In obscurin A morphant embryos at $24 \mathrm{hpf}$, talin does localize to the irregularly organized MTJs but there are regions in which laminin has been deposited where talin has not yet accumulated (arrow). (e), (f) In control and obscurin A morphant embryos, talin localizes overlying the $Z$ disk (arrowheads) although there are regions of diffuse talin localization in the morphant (asterisk) that were not noted in the control. (g) In newly forming myofibrils talin localizes along the myofibril (arrowhead) before achieving a striated pattern overlying the Z disk. No talin localization was noted overlying the mid-A-band. (h), (k) Like talin, vinculin localized to the somite boundary/MTJ ((h): arrowhead) at $24 \mathrm{hpf}$ in both control (h) and morphant (k) embryos. (i), (l), (j), (m) By $48 \mathrm{hpf}$, there is progressive accumulation of vinculin at the MTJ in control (i) and morphant (l) embryos with nearly exclusive localization to the MTJ by $72 \mathrm{hpf}$ in both control (j) and morphant (m) embryos. Scale bars are $50 \mu \mathrm{m}$. Panels (e) $-(\mathrm{g})$ are $3 \mathrm{x}$ magnifications of the corresponding regions of $(\mathrm{a}),(\mathrm{c})$, and $(\mathrm{d})$.

these two cellular compartments during early myofibril assembly. If obscurin A was required for this process, then its depletion might result in aberrant patterning of the sarcolemma overlying the myofibril and reduced association of the myofibril with the membrane. Examination of transverse sections of zebrafish skeletal muscle by transmission electron microscopy revealed that, as in control embryos (Figure 8(a)), new myofibril assembly in obscurin-Adepleted embryos occurred adjacent to the sarcolemma, preferentially at sites where three cells were in direct contact
(Figure 8(b)). However, there appeared to be reduced association of the myofibrils with the membrane, as new myofibril bundles were noted at a distance from the sarcolemma (Figures 8(c) and 8(d)). Patterning of internal membranes was also affected, as the sarcoplasmic reticulum was deficient, irregularly patterned and not consistently closely associated with the myofibrils in obscurin-depleted embryos.

Similar findings were noted on longitudinal sections. In control embryos, new myofibril assembly occurred in the subsarcolemmal space. Interestingly, there was a relatively 
Control MO

$\alpha$-dystroglycan, $24 \mathrm{hpf}$

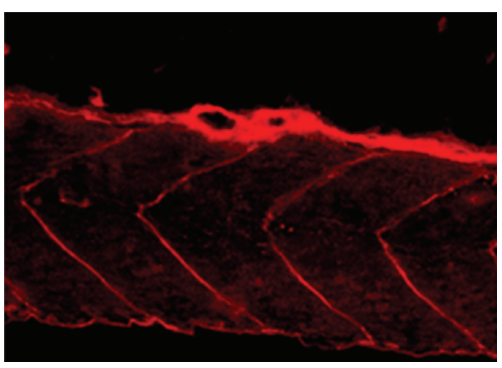

(a)

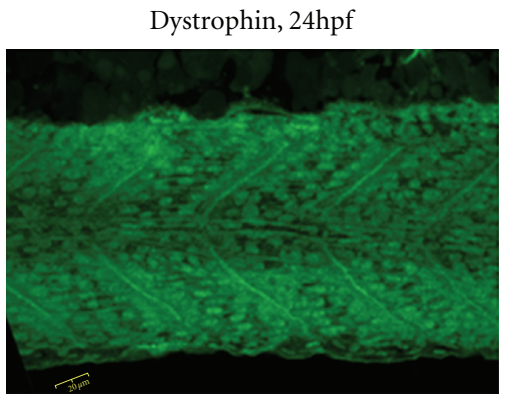

(c)
Obscurin morphant

$\alpha$-dystroglycan, 24 hpf

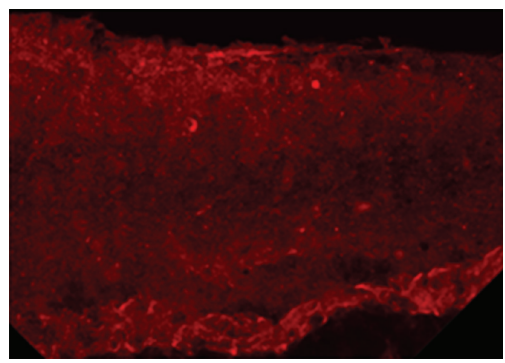

(b)

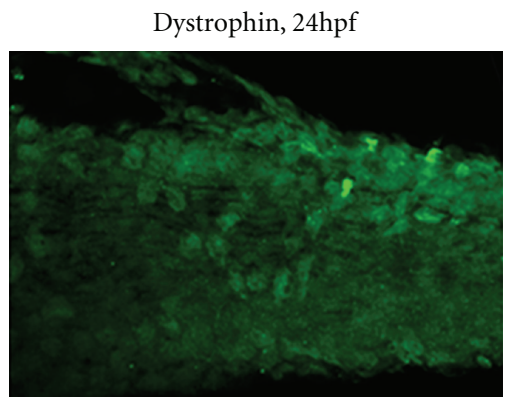

(d)

Figure 7: Obscurin depletion leads to marked reduction in localization of $\alpha$-dystroglycan and dystrophin to the myotendinous junction. $\alpha$ dystroglycan ( $\alpha$-DG) (a), (b) and dystrophin (c), (d) are abundant at the myotendinous junctions (MTJ) in control (a), (c) but not obscurin morphant embryos (b), (d). Note that in the obscurin-depleted embryos, $\alpha$-DG and dystrophin are not noted even in the disorganized MTJs of the targeted embryos suggesting a specific destabilization of dystrophin-associated glycoprotein complex (DGC) in response to decreased obscurin expression.

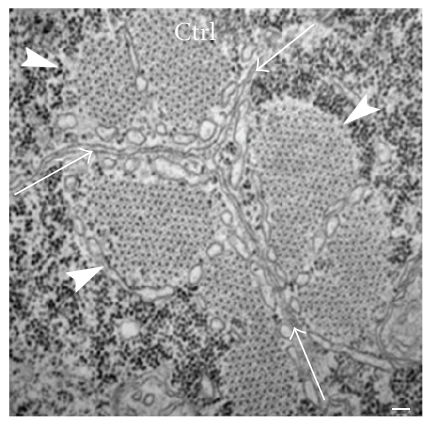

(a)

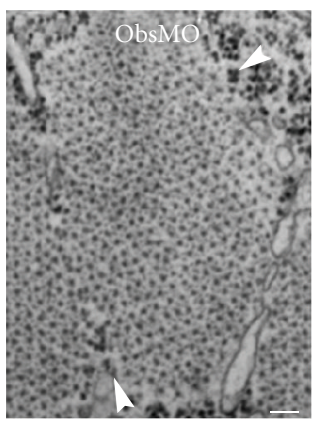

(b)

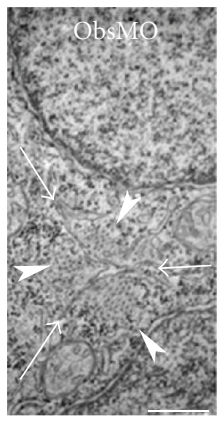

(c)

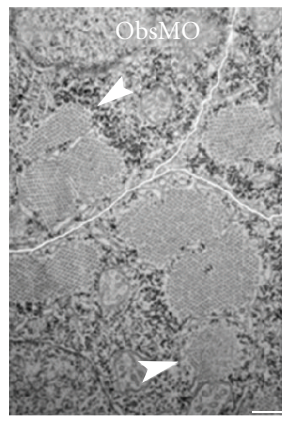

(d)

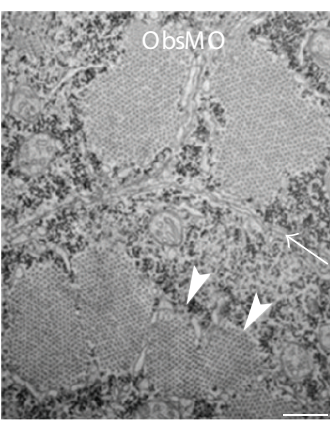

(e)

Figure 8: Obscurin depletion affects membrane-myofibril interactions. Skeletal muscle from 24 hpf zebrafish embryos were evaluated by transmission electron microscopy. (a) New myofibril assembly (arrowheads) occurs along the sarcolemma (arrows), preferentially at the intersection of three myocytes. Note the well-formed sarcoplasmic reticulum that encircles the new myofibrils. (b)-(e) In obscurin A morphant embryos, the sarcoplasmic reticulum is less well developed and less closely associated with the myofibril (b). Myofibril assembly still preferentially occurs at the sarcolemmal intersections ((c): arrowheads). However, frequent "internal myofibrils" form ((d): arrowheads) and distances between superficial myofibrils and the membrane increase ((e): arrowheads). Scale bars are $100 \mathrm{~nm}$ (a), (b) and $500 \mathrm{~nm}$ (c), (d).

consistent relationship between the superficial myofibrils of two adjacent cells. Although the distance from the myofibril to the membrane was variable, the distance between myofibrils in adjoining myocytes was within a relatively narrow range in control embryos (Figures 9(a) and 10(a)). In obscurin-depleted embryos, the relationship was much more variable (Figures 9(b)-9(d)). By $72 \mathrm{hpf,} \mathrm{myofibrils} \mathrm{filled}$ the sarcoplasm and the distance between the superficial myofibrils in adjoining cells had decreased in both control and obscurin-depleted embryos, although remained more variable in obscurin-depleted embryos (Figure 10(b)).

The coordination of myofibril assembly across myocytes included a consistent relationship between the level of the $\mathrm{Z}$ disks. While the myofibrils were not aligned precisely in 


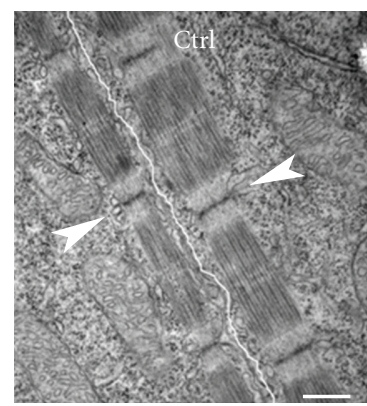

(a)

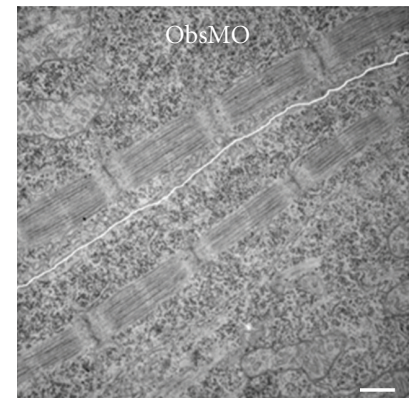

(b)

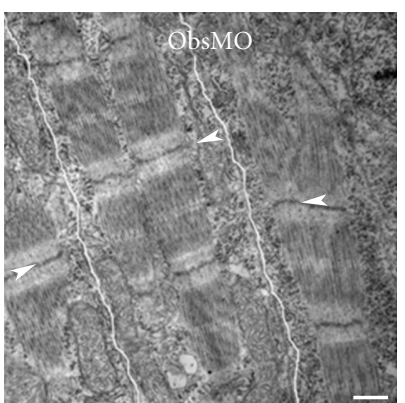

(c)

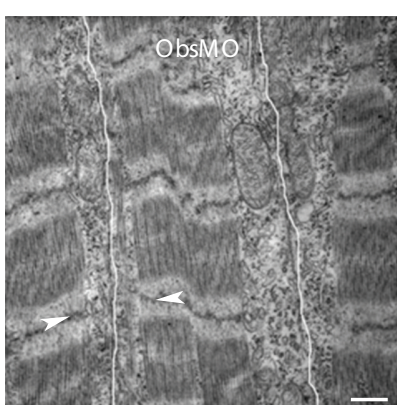

(d)

FIGURE 9: New myofibril assembly occurs along the sarcolemma with a consistent relationship between myofibrils in adjacent myocytes. Skeletal muscles from $20 \mathrm{hpf}$ zebrafish embryos were evaluated by transmission electron microscopy. (a) In control (Ctrl) embryos, new myofibril assembly occurs along the sarcolemma (white line) with a slight offset in the alignment of the $\mathrm{Z}$ disks (arrowheads). (b)-(d) In obscurin morphant (ObsMO) embryos, myofibril assembly continues to occur adjacent to the sarcolemma (white lines) but there is a greater distance and a greater variability in the distance between superficial myofibrils in adjacent cells. There was also a greater variability in the alignment of $\mathrm{Z}$ disks across adjacent myocytes, ranging from very offset ((c): arrowheads) to nearly precisely aligned ((d): arrowheads). Scale bars are $500 \mathrm{~nm}$.

register, the offset in the level of $\mathrm{Z}$ disks was within a relatively narrow range in control embryos but much more variable in obscurin-depleted muscle (Figures 9(a) and 10(c)). By $72 \mathrm{hpf}$, the $\mathrm{Z}$ disk offset had decreased in both control and obscurin-depleted embryos but remained more variable in the setting of diminished obscurin expression (Figure 10(d)).

Impairment of the coordination of myofibrillar architecture across myocytes also resulted in an inconsistent relationship between the axis of contraction in adjacent myocytes (Figure 11). In control embryos, the axis of contraction in adjacent myocytes was essentially parallel; the difference in axis of orientation between two adjacent myocytes (a myocyte pair) was $1.7 \pm 1.8^{\circ}$ in control embryos and 7.6 $\pm 7.7^{\circ}$ in obscurin depleted embryos $(t$-test: $P<0.01$ ). The impaired myocyte-myocyte relationships resulted in significant myocyte and myofibril disorganization across the muscle unit (Figures 11(b) and 11(c)).

\section{Discussion}

The assembly of new myofibrils within myocytes and the organization of the extracellular matrix surrounding the myocyte are dynamic and closely interrelated processes. Using a morphant phenotype with impaired MTJ formation and myofibril disarray, we examined the relationship between these processes during skeletal muscle development. We demonstrated that the organization of skeletal muscle during development is a highly orchestrated process that involves both outside-in and inside-out signals between the cell and the extracellular environment. Outside-in signals from the extracellular environment result in changes to myocyte morphology and promote new myofibril assembly; in return, inside-out signals organize the extracellular matrix and coordinate the assembly and organization of myofibrils across cells and tissues. Obscurin A appears to have an important role in these processes in zebrafish skeletal muscle, potentially mediating interactions between integrins and the ECM that regulate transmembrane communication and translate internal cellular structure into tissue organization.
Obscurin A is expressed early in the development, before somitogenesis begins, and our data indicate that it is required for the initial somite boundary morphogenesis. In the developing myotome, it initially localizes to the future somite boundary before localizing to newly assembling myofibrils that is form in the subsarcolemmal space. Expression patterns of MyoD, Notch5, and Notch6 indicate that loss of obscurin A disrupts somite segmentation but does not affect myotome specification or myoblast commitment. Colocalization of integrins and obscurin $\mathrm{A}$ at the future somite boundary, where myofibril assembly initiates, and along the sarcolemma, where the initiation and propagation of new myofibril assembly occurs, suggests that there may be an important relationships between integrins and obscurin A in promoting new myofibril assembly and organization. It has been proposed that integrin adhesion complexes along the lateral sarcolemma help nucleate new myofibril assembly. These early integrin-myofibril connections have been termed "protocostameres," since they will ultimately develop into the multicomponent linkage complexes characteristic of mature costameres [27]. Integrin signaling may be capable of coordinating the assembly process in neighboring cells, as suggested by the consistent relationship of $\mathrm{Z}$ disks on either side of apposed sarcolemmal membranes very early in myofibrillogenesis. A coordinated patterning of the newly assembling myofibrils in neighboring cells mediated by integrin transmembrane contacts within the sarcolemma would account for consistent relationship of the sarcomeres from one myocyte to the next. The offset of the $\mathrm{Z}$ disks in adjacent myocytes was more evident early in development and could not be correlated with distance to the MTJ indicating that it was more likely to be an inherent property of the myocyte. The reason for the offset and the molecular mechanism that supports it is not known but integrins may have a role since transinhibition by integrins have been demonstrated to inhibit integrin clustering on neighboring cells [17]. This transinhibition may inhibit integrin clustering overlying the $\mathrm{Z}$ disk if it is at exactly the same level as the superficial $\mathrm{Z}$ disk of the myofibril in the neighboring myocyte. 


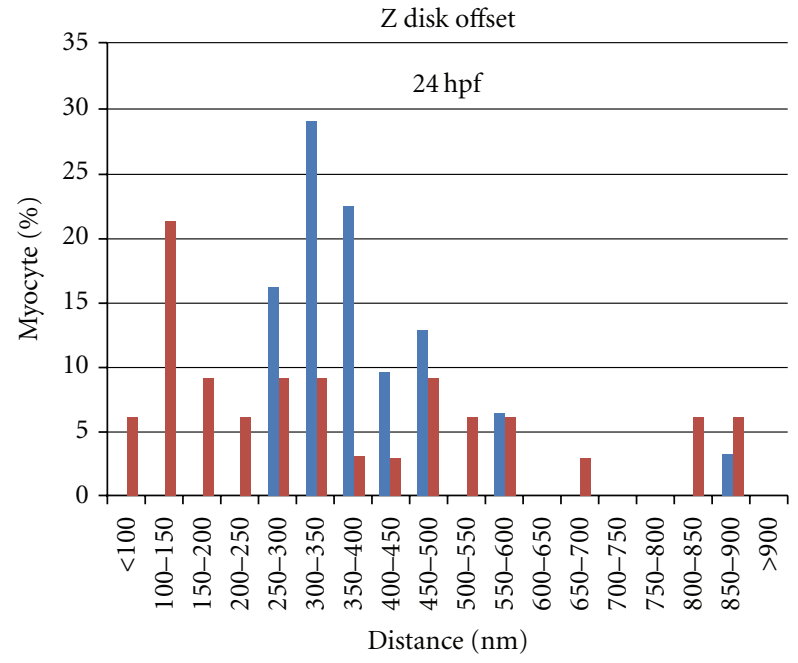

(a)

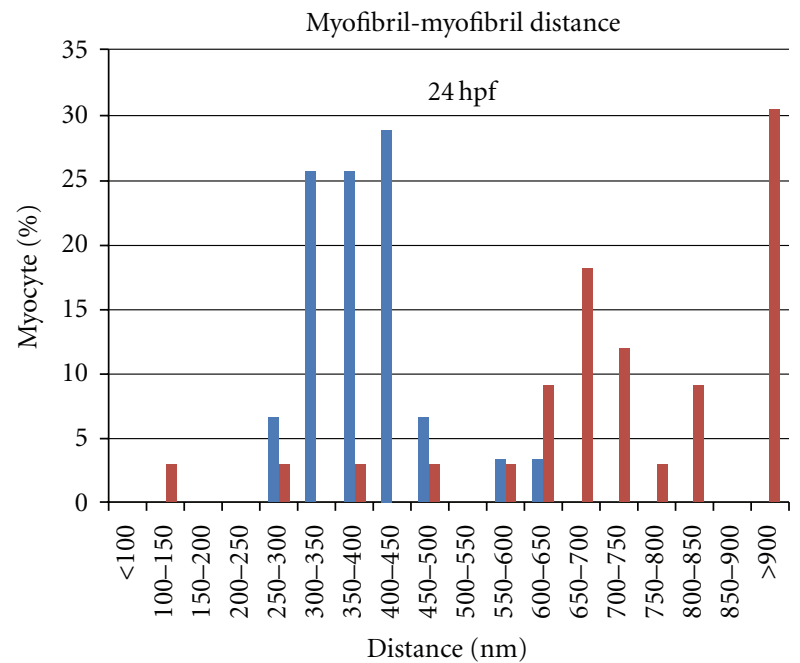

Control

ObsMO

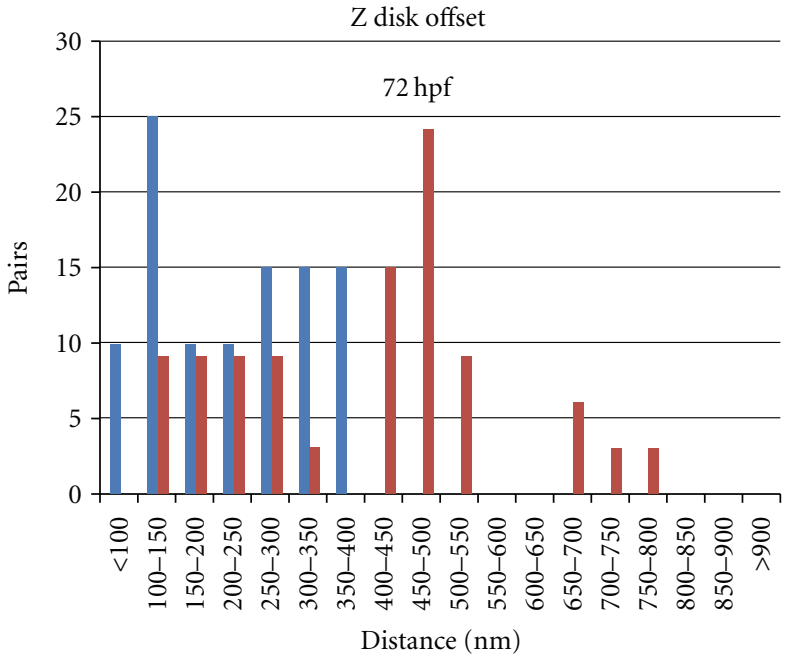

(b)

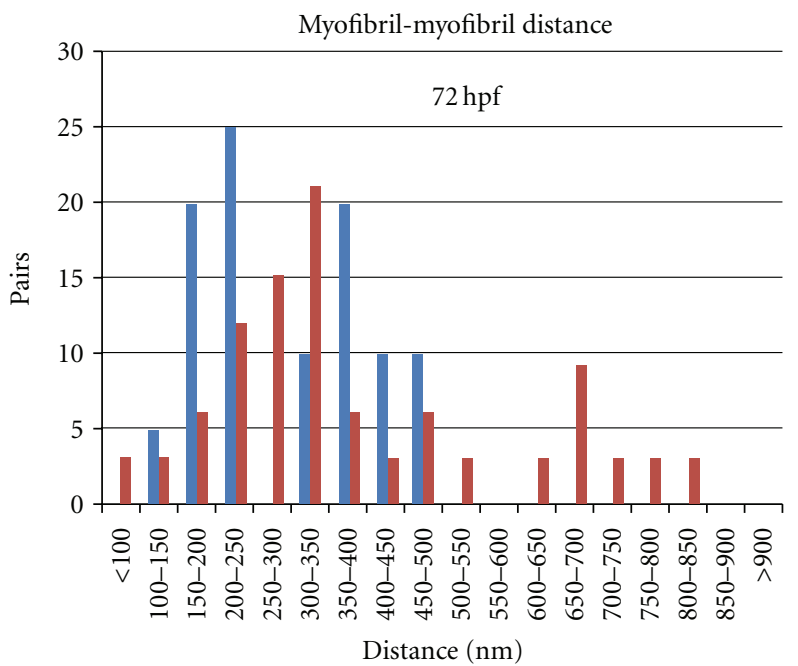

Control

ObsMO

(c)

(d)

Figure 10: Obscurin depletion results in greater variability in the relationship between superficial myofibrils in adjacent myocytes. (a) At $24 \mathrm{hpf}$, obscurin morphant embryos (red bars) demonstrate a greater variability in $\mathrm{Z}$ disk alignment than control embryos (blue bars). Note that in the majority of myocyte pairs evaluated, the superficial myofibrils demonstrate a $Z$ disk offset of approximately $250-500 \mathrm{~nm}$ in control embryos. (b) By $72 \mathrm{hpf}$, the $\mathrm{Z}$ disk offset has been significantly reduced in control embryos but was still quite variable in obscurin morphant embryos. (c) Similarly, at $24 \mathrm{hpf}$ in control embryos (blue bars), there was a consistent distance between the superficial myofibrils in adjacent myocytes. However, in obscurin morphant embryos, the distance is significantly greater and more variable. (d) By 72 hpf, the distance between superficial myofibrils has been reduced in control and obscurin morphant embryos but remains more variable in the obscurin morphants.

The potential role of obscurin A in regulating integrinbased adhesion complexes is supported by studies on Unc89. C. elegans mutants lacking Unc-89 have defects in $M$ band assembly, thick filament organization and locomotion (leading to its classification as one of the uncoordinated family of proteins) $[28,29]$. It has been noted to participate in the recruitment of actopaxin, a binding partner of the adherens junction adapter protein paxillin [30], to the sarcolemma [31]. More recently, the kinase domains of Unc89 were determined to interact with SCPL- $1[32,33]$ which in turn has been demonstrated to complex with Lim-9 to interact with Unc-96 and Unc-97 (PINCH) [34], components of a cytoskeletal network that links integrin adhesion complex assembly [35] to thick filament organization [34] in C. elegans. That these integrin-based adhesion complexes participate in the transmission of force from the sarcomere 


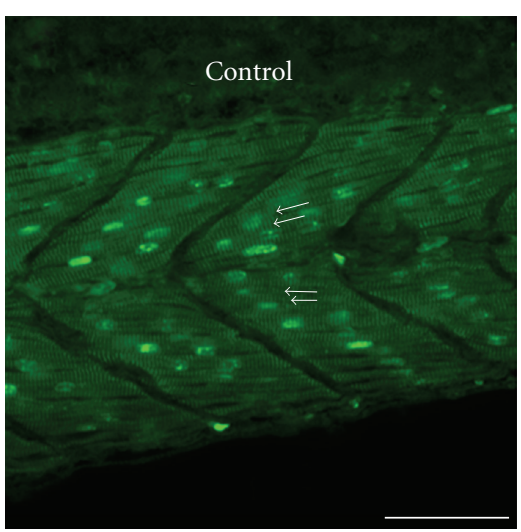

(a)

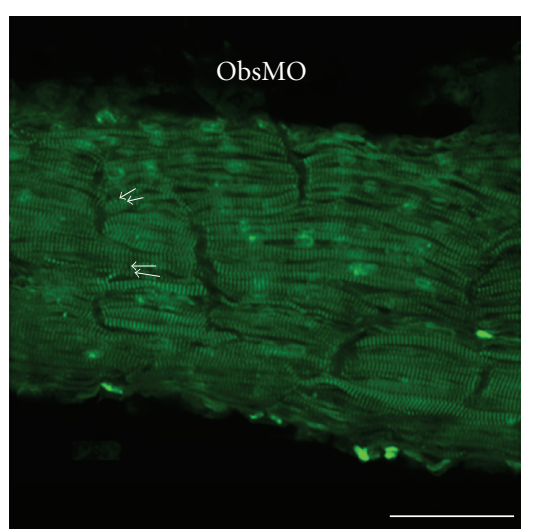

(b)

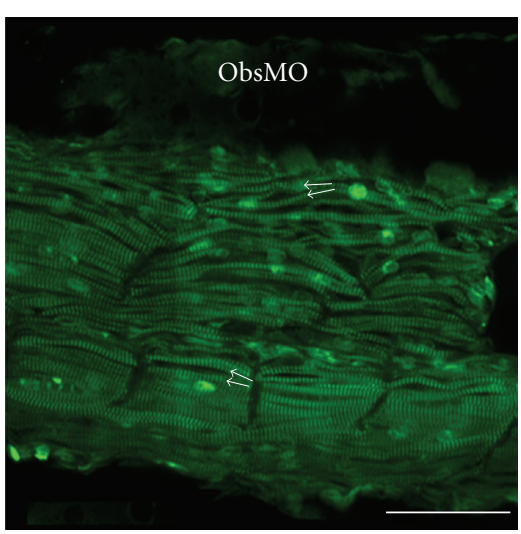

(c)

Figure 11: Obscurin depletion results in diminished alignment of myofibrils in adjacent myocytes. (a) At 48 hpf, myofibrils in adjacent myocytes are aligned (arrows) along a nearly identical axis of contraction. (b), (c) In obscurin morphant embryos, there is a loss of the precise parallel alignment (arrows) of myofibrils in adjacent myocytes. Scale bars are $50 \mu \mathrm{m}$.

to the extracellular environment to elicit movement has been supported by the genetic characterization of C.elegans mutant embryos, known as pat (for paralyzed arrested at twofold) mutants, with a paralyzed phenotype [36]. Mutations of beta integrin (pat-3), alpha integrin (pat-2), integrin-linked kinase (pat-4), and actopaxin (pat-6) all impair the assembly of integrin adhesion complexes and all result in a paralyzed phenotype [37]. In C.elegans, integrin complexes are found at the cytoplasmic interface of the dense bodies (corresponding to vertebrate $\mathrm{Z}$ disks) and the $\mathrm{M}$ bands [38]. In vertebrates, integrins are localized to the myotendinous junctions and to the costameres, sites of force transmission and signaling between the myocyte and the ECM. It is important to note that, although obscurin is primarily considered an $\mathrm{M}$ band protein, it localizes overlying the $\mathrm{Z}$ disk early in myofibril assembly [14] and some isoforms are noted at the $\mathrm{Z}$ disk or $\mathrm{Z} / \mathrm{I}$ junction in mature myofibrils [39].

The importance of the integrin-fibronectin attachments to myofibril organization was demonstrated in two zebrafish models. In zebrafish embryos depleted of TGFBI, an extracellular matrix protein that binds to integrins and is expressed in response to TGF-beta signaling [16], skeletal muscle formed normal appearing MTJs and myofibrils assembled in the subsarcolemmal space but the myofibrils did not remain closely attached to the sarcolemma, and there was markedly reduced myofibril content. Similarly, zebrafish depleted of fibronectin demonstrated reduced myofibril content and organization [20] further supporting the role of integrin-mediated ECM adhesion as an important regulator of myofibril organization. The obscurin-A-depleted embryos demonstrated very similar findings, consistent with disturbance of the fibronectin-integrin-TGFBI axis. As with the TGFBI embryos, obscurin-depleted myofibrils formed but did not consistently remain closely associated with the membrane, and the resulting myocyte/myofibril disorder was reminiscent of embryos lacking fibronectin.

\section{Conclusions}

Our studies are consistent with the previously described models of new myofibril assembly which have proposed a multistep process by which myofibrils organize along the sarcolemma [40-42]. Our studies suggest that formation of new myofibrils in the subsarcolemmal space promotes coordinated organization of the myofibril, the overlying sarcolemma, and surrounding extracellular matrix. These interactions, which depend in part on obscurin, facilitate organization of myofibrils and myocytes across the muscle permitting efficient muscle contraction and striated muscle function. Such coordinate changes in myofibrillar and ECM remodeling will be important not only during development but in the adaptation of muscle (both cardiac and skeletal) to tissue injury or increases in physiologic demand. Therefore, the role of obscurin in facilitating interactions between the myofibril and the ECM through the sarcolemma indicates that it may be an important therapeutic target in preventing muscle injury and promoting muscle repair.

\section{Acknowledgments}

The authors would like to acknowledge Ashley Bieniek for her assistance with the care of the zebrafish. The work was supported by funds from the Aaron Stern Professorship (MWR) and the NIH (HL075093). We would like to thank Dr. Scott Holley and Dr. Robert Bloch for reagents and helpful comments.

\section{References}

[1] R. J. Bloch, Y. Capetanaki, A. O’Neill et al., "Costameres: repeating structures at the sarcolemma of skeletal muscle," Clinical Orthopaedics and Related Research, no. 403, pp. S203S210, 2002.

[2] J. V. Pardo, J. D. Siliciano, and S. W. Craig, "A vinculincontaining cortical lattice in skeletal muscle: transverse lattice 
elements ("costameres") mark sites of attachment between myofibrils and sarcolemma," Proceedings of the National Academy of Sciences of the United States of America, vol. 80, no. 4 I, pp. 1008-1012, 1983.

[3] E. M. McNally and P. Pytel, "Muscle diseases: the muscular dystrophies," Annual Review of Pathology, vol. 2, pp. 87-109, 2007.

[4] L. Durbin, C. Brennan, K. Shiomi et al., "Eph signaling is required for segmentation and differentiation of the somites," Genes and Development, vol. 12, no. 19, pp. 3096-3109, 1998.

[5] C. J. Snow and C. A. Henry, "Dynamic formation of microenvironments at the myotendinous junction correlates with muscle fiber morphogenesis in zebrafish," Gene Expression Patterns, vol. 9, no. 1, pp. 37-42, 2009.

[6] C. A. Henry, I. M. McNulty, W. A. Durst, S. E. Munchel, and S. L. Amacher, "Interactions between muscle fibers and segment boundaries in zebrafish," Developmental Biology, vol. 287, no. 2, pp. 346-360, 2005.

[7] B. D. Crawford, C. A. Henry, T. A. Clason, A. L. Becker, and M. B. Hille, "Activity and distribution of paxillin, focal adhesion kinase, and cadherin indicate cooperative roles during zebrafish morphogenesis," Molecular Biology of the Cell, vol. 14, no. 8, pp. 3065-3081, 2003.

[8] D. I. Bassett, R. J. Bryson-Richardson, D. F. Daggett, P. Gautier, D. G. Keenan, and P. D. Currie, "Dystrophin is required for the formation of stable muscle attachments in the zebrafish embryo," Development, vol. 130, no. 23, pp. 5851-5860, 2003.

[9] S. Ehmer, R. Herrmann, R. Bittner, and T. Voit, "Spatial distribution of $\beta$-spectrin in normal and dystrophic human skeletal muscle," Acta Neuropathologica, vol. 94, no. 3, pp. 240-246, 1997.

[10] M. O. Raeker, F. Su, S. B. Geisler et al., "Obscurin is required for the lateral alignment of striated myofibrils in zebrafish," Developmental Dynamics, vol. 235, no. 8, pp. 2018-2029, 2006.

[11] M. O. Raeker, A. N. Bieniek, A. S. Ryan, H. J. Tsai, K. M. Zahn, and M. W. Russell, "Targeted deletion of the zebrafish obscurin A RhoGEF domain affects heart, skeletal muscle and brain development," Developmental Biology, vol. 337, no. 2, pp. 432-443, 2010.

[12] P. Bagnato, V. Barone, E. Giacomello, D. Rossi, and V. Sorrentino, "Binding of an ankyrin-1 isoform to obscurin suggests a molecular link between the sarcoplasmic reticulum and myofibrils in striated muscles," Journal of Cell Biology, vol. 160, no. 2, pp. 245-253, 2003.

[13] M. W. Russell, M. O. Raeker, K. A. Korytkowski, and K. J. Sonneman, "Identification, tissue expression and chromosomal localization of human Obscurin-MLCK, a member of the titin and Dbl families of myosin light chain kinases," Gene, vol. 282, no. 1-2, pp. 237-246, 2002.

[14] P. Young, E. Ehler, and M. Gautel, "Obscurin, a giant sarcomeric Rho guanine nucleotide exchange factor protein involved in sarcomere assembly," Journal of Cell Biology, vol. 154, no. 1, pp. 123-136, 2001.

[15] Y. Yamanashi, S.-I. Fukushige, and K. Semba, "The yes-related cellular gene lyn encodes a possible tyrosine kinase similar to p56(lck)," Molecular and Cellular Biology, vol. 7, no. 1, pp. 237-243, 1987.

[16] H. R. Kim and P. W. Ingham, "The extracellular matrix protein TGFBI promotes myofibril bundling and muscle fibre growth in the zebrafish embryo," Developmental Dynamics, vol. 238, no. 1, pp. 56-65, 2009.

[17] D. Julich, A. P. Mould, E. Koper, and S. A. Holley, "Control of extracellular matrix assembly along tissue boundaries via integrin and Eph/Ephrin signaling," Development, vol. 136, no. 17, pp. 2913-2921, 2009.

[18] S. Koshida, Y. Kishimoto, H. Ustumi et al., "Integrin $\alpha 5$-dependent fibronectin accumulation for maintenance of somite boundaries in zebrafish embryos," Developmental Cell, vol. 8, no. 4, pp. 587-598, 2005.

[19] D. Julich, R. Geisler, S. A. Holley et al., "Integrin $\alpha 5$ and delta/ notch signaling have complementary spatiotemporal requirements during zebrafish somitogenesis," Developmental Cell, vol. 8, no. 4, pp. 575-586, 2005.

[20] C. J. Snow, M. T. Peterson, A. Khalil, and C. A. Henry, "Muscle development is disrupted in zebrafish embryos deficient for fibronectin," Developmental Dynamics, vol. 237, no. 9, pp. 2542-2553, 2008.

[21] M. J. Parsons, I. Campos, E. M. A. Hirst, and D. L. Stemple, "Removal of dystroglycan causes severe muscular dystrophy in zebrafish embryos," Development, vol. 129, no. 14, pp. 35053512, 2002.

[22] F. Bolanos-Jimenez, A. Bordais, M. Behra et al., "Molecular cloning and characterization of dystrophin and Dp71, two products of the Duchenne Muscular Dystrophy gene, in zebrafish," Gene, vol. 274, pp. 217-226, 2001.

[23] P. M. Bennett, A. J. Baines, M. C. Lecomte, A. M. Maggs, and J. C. Pinder, "Not just a plasma membrane protein: in cardiac muscle cells alpha-II spectrin also shows a close association with myofibrils," Journal of Muscle Research and Cell Motility, vol. 25, no. 2, pp. 119-126, 2004.

[24] A. A. Hopitzan, A. J. Baines, and E. Kordeli, "Molecular evolution of ankyrin: gain of function in vertebrates by acquisition of an obscurin/titin-binding-related domain," Molecular Biology and Evolution, vol. 23, no. 1, pp. 46-55, 2006.

[25] A. Kontrogianni-Konstantopoulos, E. M. Jones, D. B. Van Rossum, and R. J. Bloch, "Obscurin is a ligand for small ankyrin 1 in skeletal muscle," Molecular Biology of the Cell, vol. 14, no. 3, pp. 1138-1148, 2003.

[26] M. L. Bang, T. Centner, F. Fornoff et al., "The complete gene sequence of titin, expression of an unusual $\approx 700-\mathrm{kDa}$ titin isoform, and its interaction with obscurin identify a novel Zline to I-band linking system," Circulation Research, vol. 89, no. 11, pp. 1065-1072, 2001.

[27] J. C. Sparrow and F. Schock, "The initial steps of myofibril assembly: integrins pave the way," Nature Reviews Molecular Cell Biology, vol. 10, no. 4, pp. 293-298, 2009.

[28] G. M. Benian, T. L. Tinley, X. Tang, and M. Borodovsky, "The Caenorhabditis elegans gene unc-89, required for muscle Mline assembly, encodes a giant modular protein composed of Ig and signal transduction domains," Journal of Cell Biology, vol. 132 , no. 5, pp. 835-848, 1996.

[29] R. H. Waterston, J. N. Thomson, and S. Brenner, "Mutants with altered muscle structure in Caenorhabditis elegans," Developmental Biology, vol. 77, no. 2, pp. 271-302, 1980.

[30] S. N. Nikolopoulos and C. E. Turner, "Actopaxin, a new focal adhesion protein that binds paxillin LD motifs and actin and regulates cell adhesion," Journal of Cell Biology, vol. 151, no. 7, pp. 1435-1447, 2000.

[31] X. Lin, H. Qadota, D. G. Moerman, and B. D. Williams, "C. elegans PAT-6/actopaxin plays a critical role in the assembly of integrin adhesion complexes in vivo," Current Biology, vol. 13, no. 11, pp. 922-932, 2003.

[32] G. Xiong, H. Qadota, K. B. Mercer, L. A. McGaha, A. F. Oberhauser, and G. M. Benian, "A LIM-9 (FHL)/SCPL-1 (SCP) complex interacts with the C-terminal protein kinase regions of UNC-89 (obscurin) in Caenorhabditis elegans muscle," Journal of Molecular Biology, vol. 386, no. 4, pp. 976-988, 2009. 
[33] H. Qadota, L. A. McGaha, K. B. Mercer, T. J. Stark, T. M. Ferrara, and G. M. Benian, "A novel protein phosphatase is a binding partner for the protein kinase domains of UNC-89 (obscurin) in Caenorhabditis elegans," Molecular Biology of the Cell, vol. 19, no. 6, pp. 2424-2432, 2008.

[34] H. Qadota, K. B. Mercer, R. K. Miller, K. Kaibuchi, and G. M. Benian, "Two LIM domain proteins and UNC-96 link UNC-97/PINCH to myosin thick filaments in Caenorhabditis elegans muscle," Molecular Biology of the Cell, vol. 18, no. 11, pp. 4317-4326, 2007.

[35] K. R. Norman, S. Cordes, H. Qadota, P. Rahmani, and D. G. Moerman, "UNC-97/PINCH is involved in the assembly of integrin cell adhesion complexes in Caenorhabditis elegans body wall muscle," Developmental Biology, vol. 309, no. 1, pp. 45-55, 2007.

[36] B. D. Williams and R. H. Waterston, "Genes critical for muscle development and function in Caenorhabditis elegans identified through lethal mutations," Journal of Cell Biology, vol. 124, no. 4, pp. 475-490, 1994.

[37] H. Qadota and G. M. Benian, "Molecular structure of sarcomere-to-membrane attachment at M-lines in C. elegans muscle," Journal of Biomedicine and Biotechnology, vol. 2010, Article ID 864749, 9 pages, 2010.

[38] D. G. Moerman and B. D. Williams, "Sarcomere assembly in C. elegans muscle," WormBook, pp. 1-16, 2006.

[39] A. L. Bowman, A. Kontrogianni-Konstantopoulos, S. S. Hirsch et al., "Different obscurin isoforms localize to distinct sites at sarcomeres," FEBS Letters, vol. 581, no. 8, pp. 1549-1554, 2007.

[40] J. W. Sanger, J. Wang, B. Holloway, A. Du, and J. M. Sanger, "Myofibrillogenesis in skeletal muscle cells in zebrafish," Cell Motility and the Cytoskeleton, vol. 66, no. 8, pp. 556-566, 2009.

[41] A. Du, J. M. Sanger, and J. W. Sanger, "Cardiac myofibrillogenesis inside intact embryonic hearts," Developmental Biology, vol. 318, no. 2, pp. 236-246, 2008.

[42] J. W. Sanger, S. Kang, C. C. Siebrands et al., "How to build a myofibril," Journal of Muscle Research and Cell Motility, vol. 26, no. 6-8, pp. 343-354, 2005. 

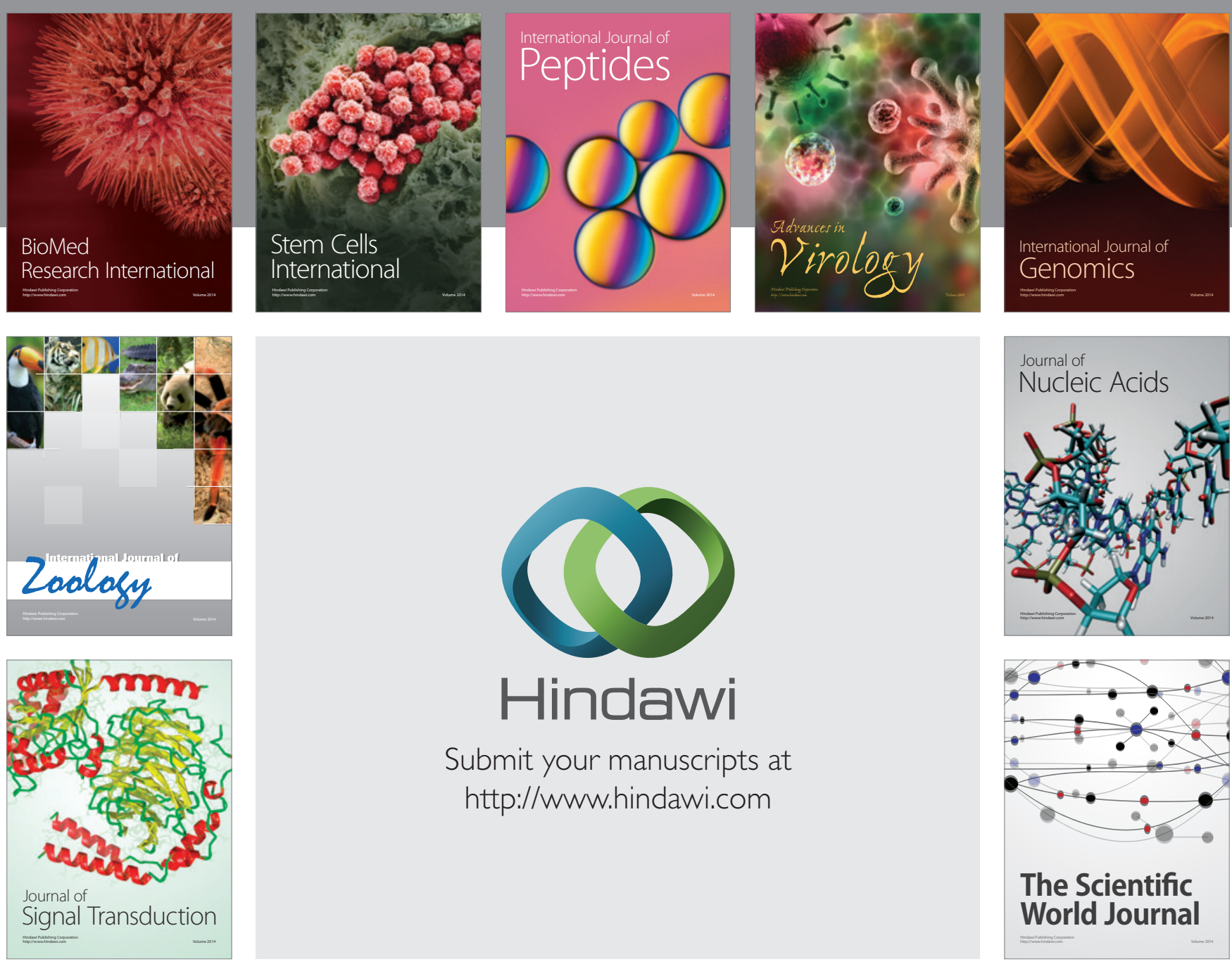

Submit your manuscripts at

http://www.hindawi.com
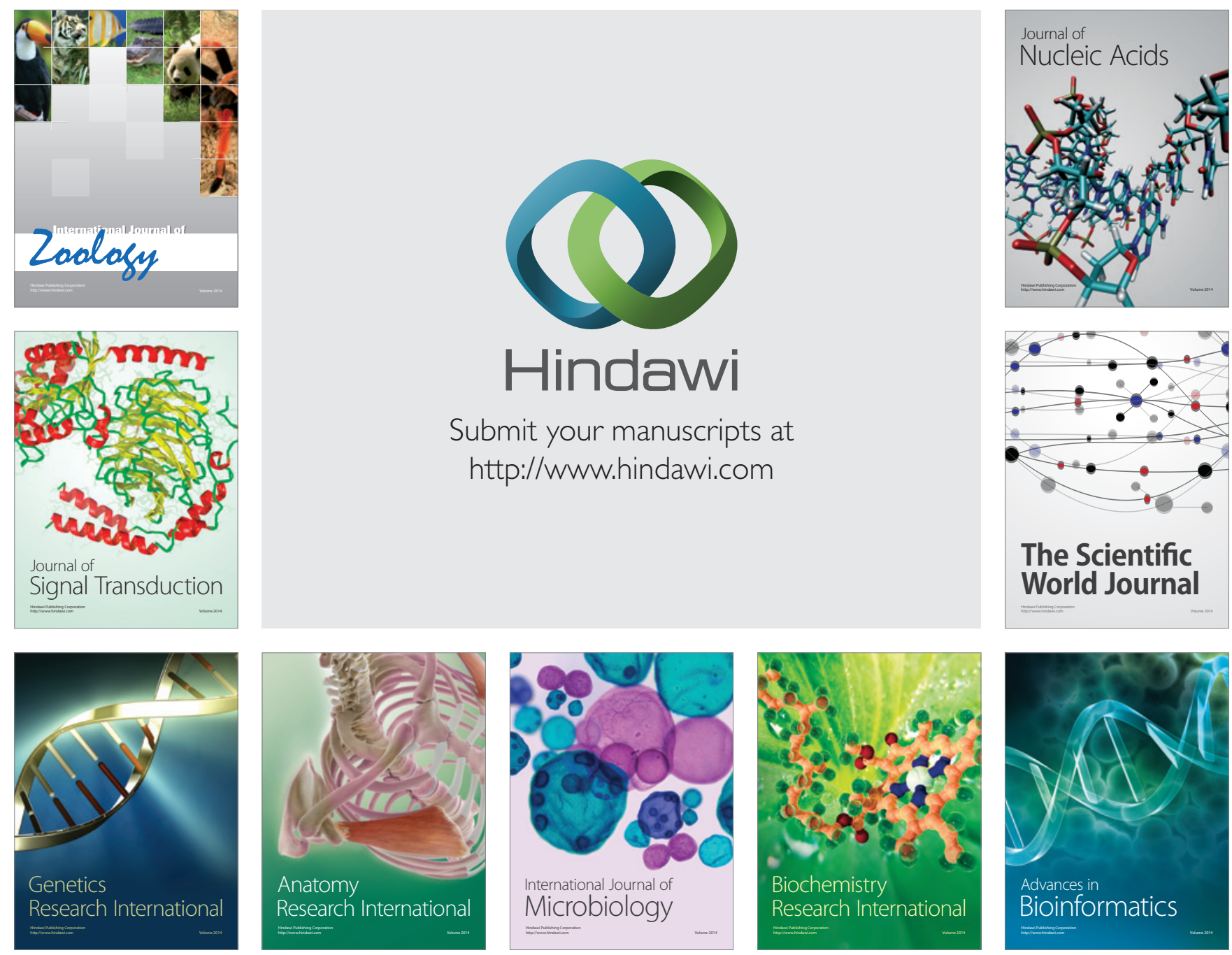

The Scientific World Journal
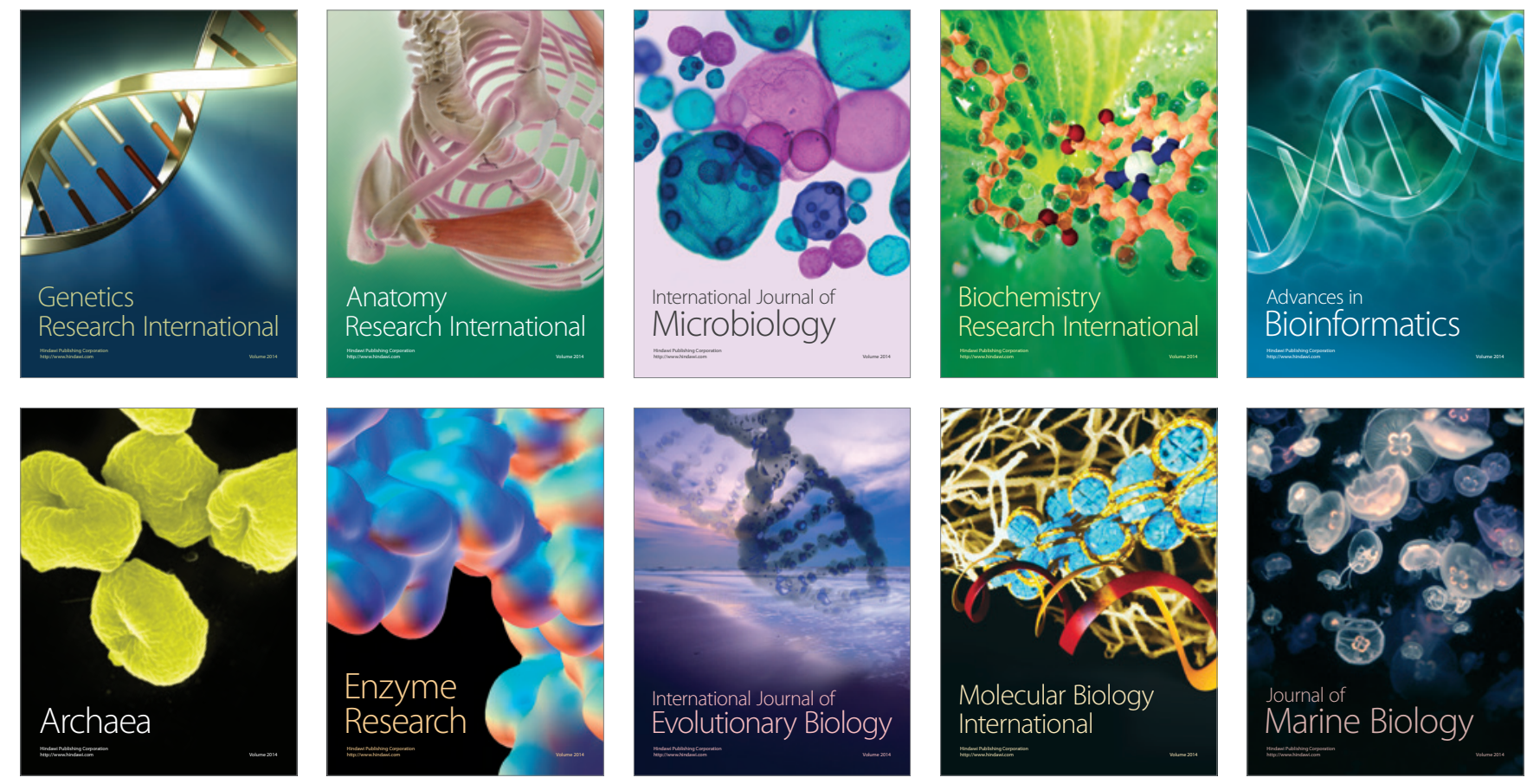